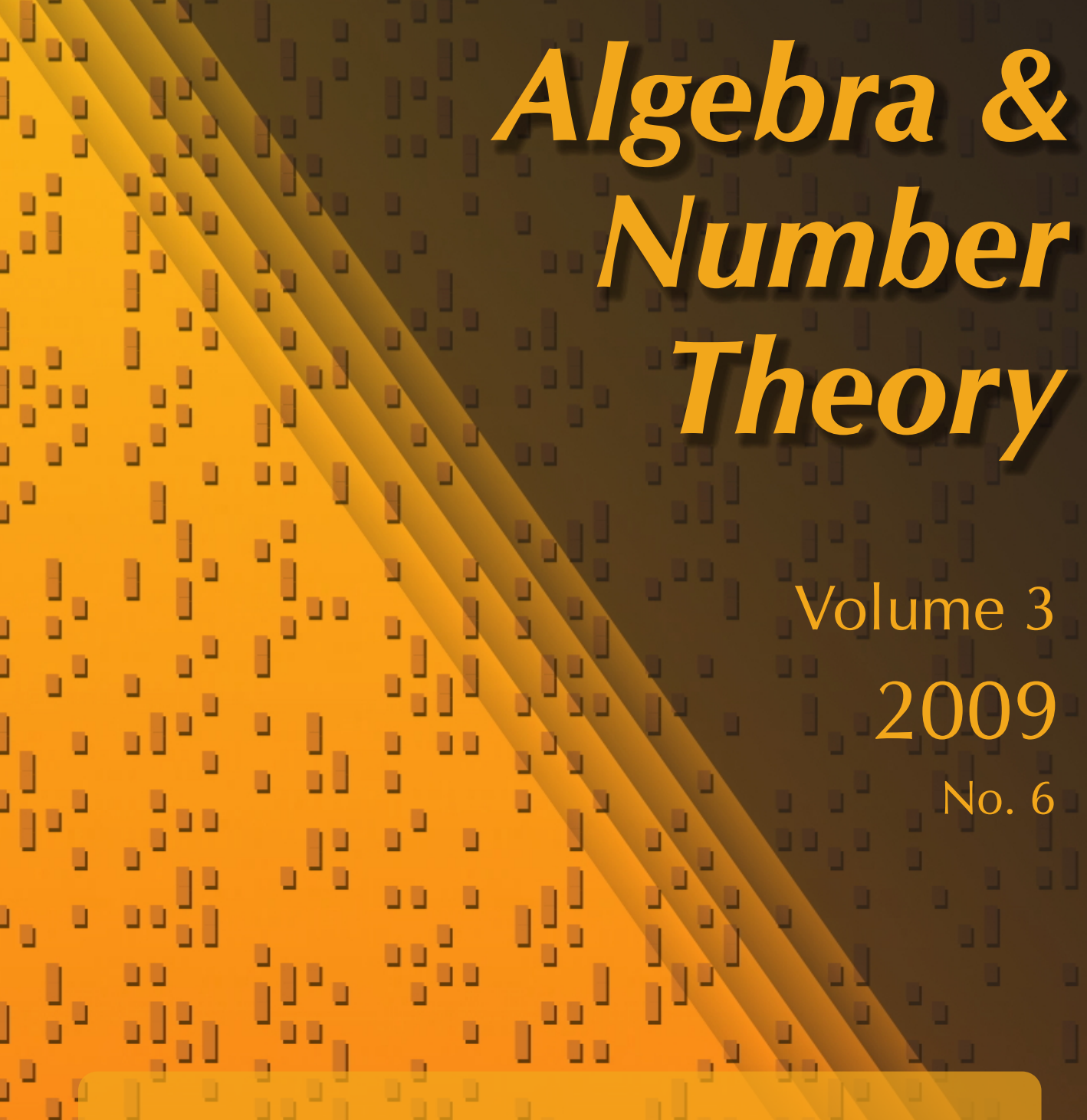




\title{
Compactified moduli of projective bundles
}

\author{
Max Lieblich
}

\begin{abstract}
We present a method for compactifying stacks of $\mathrm{PGL}_{n}$-torsors (Azumaya algebras) on algebraic spaces. In particular, when the ambient space is a smooth projective surface we use our methods to show that various moduli spaces are irreducible and carry natural virtual fundamental classes. We also prove a version of the Skolem-Noether theorem for certain algebra objects in the derived category, which allows us to give an explicit description of the boundary points in our compactified moduli problem.
\end{abstract}

1. Introduction 653

2. Notation 655

3. Generalities 656

4. Compactified moduli of $\mathrm{PGL}_{n}$-torsors: an abstract approach 667

5. An explicit description of $\mathcal{M}_{n}^{\mathscr{X}}$ : generalized Azumaya algebras 670

6. Moduli of stable $\mathrm{PGL}_{n}$-torsors on surfaces 683

Acknowledgments $\quad 693$

References $\quad 693$

\section{Introduction}

In this paper, we present a method for constructing compactified moduli of principal $\mathrm{PGL}_{n}$-bundles on an algebraic space. As a demonstration of its usefulness, we will prove the following theorem.

Irreducibility Theorem (Theorem 6.3.1). Let X be a smooth projective surface over an algebraically closed field $k$ and $n$ a positive integer which is invertible in $k$. For any cohomology class $\alpha \in \mathrm{H}^{2}\left(X, \mu_{n}\right)$, the stack of stable $\mathrm{PGL}_{n}$-torsors on $X$ with cohomology class $\alpha$ and sufficiently large $c_{2}$ is of finite type and irreducible whenever it is nonempty, and it is nonempty infinitely often.

MSC2000: primary 14D20; secondary 14D15.

Keywords: projective bundles, moduli of stable bundles, Skolem-Noether theorem, derived categories, rigidification.

The work described in this paper was partially supported at various stages by an NSF Graduate Fellowship, a Clay Liftoff Fellowship, an NSF Postdoctoral Fellowship, and NSF grant DMS-0758391. 
Here the number $c_{2}$ is meant to be the second Chern class of the adjoint vector bundle associated to a $\mathrm{PGL}_{n}$-torsor. For the definition of stability of a $\mathrm{PGL}_{n}$-torsor, we refer the reader to Definition 6.1.2 below; in characteristic 0 it is equivalent to slope-stability of the adjoint vector bundle, while in arbitrary characteristic one quantifies only over ideals in the adjoint (with respect to its natural Azumaya algebra structure).

The Irreducibility Theorem may be viewed as a zeroth-order algebraic version of results of Mrowka and Taubes (see [Taubes 1989], for instance) on the stable topology of the space of $\mathrm{PGL}_{n}$-bundles; we show that $\pi_{0}$ is a singleton. Our proof arises out of a reduction of the moduli problem to another recently studied problem: moduli of twisted sheaves. Before making a few historical remarks, let us outline the contents of the paper.

In Section 3.4 we present a general theory of twisted objects in a stack, including the resulting deformation theory and the relationship between twisted and untwisted virtual fundamental classes. In Section 4 we apply this theory to $\mathrm{PGL}_{n^{-}}$ torsors to show that the stack of twisted sheaves is naturally a cover of a compactification of the stack of $\mathrm{PGL}_{n}$-torsors.

In Section 5, we give a reinterpretation of the results of Section 4 using certain algebra objects of the derived category (generalized Azumaya algebras), with the ultimate aim being an approach to virtual fundamental classes for spaces of stable $\mathrm{PGL}_{n}$-torsors. The key result there is Theorem 5.1.5, a version of the SkolemNoether theorem for these algebra objects, which we believe should be of independent interest.

In Section 6, we specialize the whole picture to study moduli of $\mathrm{PGL}_{n}$-torsors on smooth projective surfaces. We develop the theory of stability in Section 6.1 and use the known structure theory of moduli spaces of twisted sheaves on a surface to prove the Irreducibility Theorem in Section 6.3. In Sections 6.4 and 6.5, we use the interpretation of the moduli problem in terms of generalized Azumaya algebras to produce virtual fundamental classes on moduli spaces of stable $\mathrm{PGL}_{n^{-}}$ torsors on surfaces. In Section 6.6, we record a question due to de Jong regarding potentially new numerical invariants for division algebras over function fields of surfaces arising out the virtual fundamental classes constructed in Section 6.5.

Historical remarks. As has become clear in the history of algebraic geometry, a propitious choice of compactification of a moduli problem can lead to concrete results about the original (usually open) subproblem which is being compactified. Thus, Deligne and Mumford proved that $\mu_{g}$ is irreducible by embedding it as an open substack of $\bar{M}_{g}$ and connecting points by first degenerating them to the boundary. Similarly, O'Grady approached the moduli of semistable vector bundles on a surface by considering the larger space of semistable torsion free sheaves and 
showing that the boundary admits a stratification by spaces fibered over moduli of stable vector bundles with smaller $c_{2}$. Combining this inductive structure with delicate numerical estimates allowed him to prove that the spaces of semistable vector bundles with sufficiently large $c_{2}$ are irreducible. (This is very beautifully explained in Chapter 9 of [Huybrechts and Lehn 1997].) The similarity between the Irreducibility Theorem above and O'Grady's results for stable sheaves is traceable to the fact that our compactification is closely related to the space of twisted sheaves, so that we get a similar inductive structure on the moduli problem from the geometry of its boundary.

Various attempts have been made at constructing compactified moduli spaces of $G$-torsors for arbitrary (reductive) groups $G$ (the reader can consult [Gómez and Sols 2005; Hyeon 2002; Langer 2005; Schmitt 2002 2004] for a sampling of moduli problems and techniques). Of course, when $G=\mathrm{GL}_{n}$, one can take torsion free sheaves, and when $G=\mathrm{SL}_{n}$, one can take torsion-free sheaves with a trivialized determinant. In the existing literature, most compactifications proceed (at least in the case where the center of $G$ is trivial) essentially by encoding a degeneration of a principal $G$-bundle in a degeneration of its adjoint bundle to a torsion-free sheaf along with data which remember the principal $G$-bundle structure over the open subspace on which the degenerate sheaf is locally free.

We show how one can analyze the case $G=\mathrm{PGL}_{n}$ using more subtle methods, which roughly amount to allowing a principal bundle to degnerate by degenerating its associated adjoint bundle to an object of the derived category (rather than simply a torsion-free sheaf). By controlling the nature of these derived objects, we arrive at a compact moduli stack whose geometry is as tightly controlled as that of the stack of $\mathrm{SL}_{n}$-bundles. This "tight control" is formalized precisely by the covering using twisted sheaves.

\section{Notation}

All stacks will be stacks in groupoids. Thus, given an algebraic structure such as a torsion-free sheaf, the stack of objects with that structure will be assumed to keep track only of isomorphisms.

Given a geometric morphism $f: X \rightarrow S$ of topoi and a stack $\mathscr{Y}$ on $X, f_{*} \mathscr{Y}$ will denote the stack on $S$ whose sections over an object $T \in S$ are the sections of $\mathscr{Y}$ over $\pi^{-1} T \in X$. We will often write $T \rightarrow S$ for the map to the final object of $S$ and we will often use $X \times{ }_{S} T$ to denote the object $f^{-1}(T)$. Most of the topoi we encounter will be the usual étale or fppf topos of an algebraic space or stack, but we do include a few which are slightly less conventional, such as the relative small étale topos of Section 3.2. 
A stack over an algebraic space will be called quasiproper if it satisfies the existence part of the valuative criterion of properness over discrete valuation rings (allowing finite extensions, as is usually required for algebraic stacks). Given a Deligne-Mumford stack $\mathcal{M}$ with a coarse moduli space, we will let $\mathcal{M}^{\text {mod }}$ denote the coarse space. Given a moduli space (stack) $M$ of sheaves on a proper algebraic space $X$, we will let $M^{\text {lf }}$ denote the open subspace parametrizing locally free sheaves.

The notation $(\mathcal{O}, \mathfrak{m}, \kappa)$ will mean that $\mathcal{O}$ is a local ring with maximal ideal $\mathfrak{m}$ and residue field $\kappa$.

\section{Generalities}

Throughout this section, we fix a geometric morphism of ringed topoi $f: X \rightarrow S$. (In various subsections, there will be additional hypotheses on the nature of $X, S$, or $f$, but the notation will remain unchanged.)

3.1. Stacks of sheaves. There are various types of sheaves which will be important for us. We recall important definitions and set notations in this section. Let $Z$ be an algebraic space and $\mathscr{F}_{\mathrm{F}}$ a quasicoherent sheaf of finite presentation on $Z$.

The sheaf $\mathscr{F}$ is

(1) perfect if its image in $\mathbf{D}\left(\mathrm{O}_{Z}\right)$ is a perfect complex;

(2) pure if for every geometric point $z \rightarrow Z$ the stalk $\mathscr{F}_{z}$ (which is a module over the local ring $\mathrm{O}_{z, Z}^{\mathrm{hs}}$ ) has no embedded primes;

(3) totally supported if the natural map $\mathrm{O}_{Z} \rightarrow \mathscr{E} d(\mathscr{F})$ is injective;

(4) totally pure if it is pure and totally supported.

The key property of perfect sheaves for us will be the fact that one can form the determinant of any such sheaf. The reader is referred to [Knudsen and Mumford 1976] for the construction and basic facts.

It is clear that all of these properties are local in the étale topology on $Z$ (in the sense that they hold on $Z$ if and only if they hold on an étale cover). Thus, we can define various stacks on the small étale site of $Z$. We will write

(1) $\mathscr{T}_{Z}$ for the stack of totally supported sheaves;

(2) $\mathscr{T}_{Z}^{\text {parf }}$ for the stack of perfect totally supported sheaves;

(3) $\mathscr{P}_{Z}$ for the stack of pure sheaves;

(4) $\mathscr{P}_{Z}^{\text {parf }}$ for the stack of perfect pure sheaves;

(5) if $\mathcal{M}$ denotes any of the preceding stacks, we will use $\mathcal{M}(n)$ to denote the substack parametrizing sheaves with rank $n$ at each maximal point of $Z$.

In particular, if $n>0$ then $\mathscr{P}_{Z}^{\text {parf }}(n)$ parametrizes perfect totally pure sheaves. 
3.2. The relative small étale site. We recall a few pieces of pure nonsense that will help us apply the techniques of Section 3.4 below to study moduli problems. In this section, we assume that $f$ is a morphism of algebraic spaces.

Definition 3.2.1. The relative small étale site of $X / S$ is the site whose underlying category consists of pairs $(U, T)$ with $T \rightarrow S$ a morphism and $U \rightarrow X \times{ }_{S} T$ an étale morphism. A morphism $(U, T) \rightarrow\left(U^{\prime}, T^{\prime}\right)$ is an $S$-morphism $T \rightarrow T^{\prime}$ and an $T$ morphism $U \rightarrow U^{\prime} \times_{T}^{\prime} T$. A covering is a collection of maps $\left\{\left(V_{i}, T\right)\right\} \rightarrow\{(U, T)\}$ such that $V_{i} \rightarrow U$ form a covering.

We will denote the topos of sheaves on the relative small étale site by $X_{\text {rét. }}$ There is an obvious geometric morphism of topoi $X_{\text {rét }} \rightarrow T_{\text {ÉT }}$. (In fact, $X_{\text {rét }}$ is just the "total space" of a fibered topos over $T_{\text {ÉT }}$ whose fiber over $T \rightarrow S$ is just the small étale topos of $X \times{ }_{S} T$.)

The relative small étale topos is naturally suited to studying moduli of $T$-flat sheaves on $X$ (as pushforwards of $X$-stacks).

Proposition 3.2.2. Pullback defines a natural equivalence of the category of quasicoherent sheaves on $X$ with the category of quasicoherent sheaves on $X_{\text {rét. }}$ Moreover,

(1) there is a stack $\mathscr{C}_{X / S} \rightarrow X_{\text {rét }}$ whose sections over $(U, T)$ parametrize quasicoherent sheaves on $U$ which are $T$-flat and which are locally of finite presentation;

(2) if $\mathcal{M}$ denotes any of the stacks from Section 3.1, there is a substack $\mathcal{M}_{X / S} \subset$ $\mathscr{C}_{X / S}$ whose sections over $(U, T)$ are $T$-flat quasicoherent sheaves $\mathscr{F}$ of finite presentation on $U$ such that for each geometric point $t \rightarrow T$, the restriction $\mathscr{F}_{t}$ lies in $\mathcal{M}_{U_{t}}$;

(3) for each $M$, there is a substack $\mathcal{M}_{X / S}^{\mathrm{parf}} \subset \mathcal{M}_{X / S}$ parametrizing $\mathscr{F}_{\text {such }}$ that each $\mathscr{F}_{t}$ is perfect;

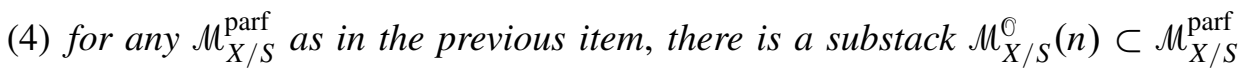
parametrizing sheaves $\mathscr{F}$ such that each fiber $\mathscr{F}_{t}$ has rank $n$ at each maximal point of $U_{t}$, along with a global trivialization $\operatorname{det} \mathscr{F} \stackrel{\sim}{\rightarrow} \mathrm{O}_{U}$.

In the last item, we implicitly use the standard fact that a quasicoherent sheaf of finite presentation which is flat over the base and perfect on each geometric fiber is perfect. As an example, we have that $\mathscr{P}_{X / S}^{\mathbb{O}}(n)$ denotes the stack on $X_{\text {rét }}$ whose objects over $(U, T)$ are pairs $(\mathscr{F}, \delta)$ with $\mathscr{F}$ perfect and $T$-flat, $\delta: \operatorname{det} \mathscr{F} \stackrel{\sim}{\rightarrow} \mathcal{O}_{U}$ an isomorphism, and such that for each geometric point $t \rightarrow T$, the sheaf $\mathscr{F}_{t}$ has rank $n$ at each maximal point of $U_{t}$.

The proof of Proposition 3.2.2 is essentially a sequence of tautologies and is omitted. Note that we cannot make any claims about algebraicity of $\mathscr{C}, \mathcal{T}$, or 
$\mathscr{P}$ because such a statement is meaningless for stacks on $X_{\text {rét }}$. However, when $f: X \rightarrow S$ is a proper morphism of finite presentation between algebraic spaces, it is of course standard that the pushforward of $\mathscr{C}_{X / S}$ to $S_{\text {ÉT }}$ is an algebraic stack (and

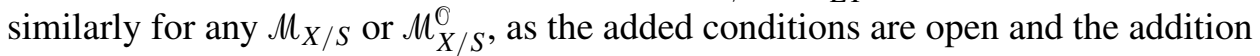
of a trivialization of the determinant is algebraic).

The following lemma will be useful later.

Lemma 3.2.3. Suppose $f: Y \rightarrow Z$ is a flat morphism of locally Noetherian schemes and $\mathscr{F}_{\mathrm{F}}$ is a $Z$-flat coherent sheaf on $Y$. If the restriction of $\mathscr{F}_{\mathrm{F}}$ to every fiber of $f$ is totally supported, then $\mathscr{F}$ is totally supported on $Y$.

Proof. We may assume that $X=\operatorname{Spec} B$ and $S=\operatorname{Spec} A$ are local schemes and that $f$ is the map associated to a local homomorphism $\varphi: A \rightarrow B$. Write $F$ for the stalk of $\mathscr{F}$ at the closed point of $B$. Choosing generators $x_{1}, \ldots, x_{n}$ for $F$, we find a surjection $B^{n} \rightarrow F$ which yields an injection $\operatorname{End}(F) \hookrightarrow F^{n}$. The composition of this injection with the natural inclusion of $B$ sends $1 \in B$ to the $n$-tuple $\left(x_{1}, \ldots, x_{n}\right) \in F^{n}$. We will show that this map $l: B \rightarrow F^{n}$ is an injection. Note that $l$ respects base change in the sense that for any $A$-algebra $C, l \otimes_{A} C$ is the map corresponding to the composition $C \rightarrow \operatorname{End}_{C}\left(F \otimes_{A} C\right) \rightarrow\left(F \otimes_{A} C\right)^{n}$. As the right-hand map in that sequence is always an injection, we find that the left-hand map is an injection if and only if $\iota \otimes_{A} C$ is an injection.

We proceed by "infinitesimal induction" relative to $A$, i.e., we write $A$ with the $\mathfrak{m}_{A}$-adic topology as an inverse limit of small extensions $\left\{A_{m}\right\}$ with $A_{0}=k(A)$, the residue field of $A$. We will show that $\lim _{\leftarrow} \imath_{m}: \widehat{B} \rightarrow \widehat{F}^{n}$ is an injection. Krull's theorem and the obvious compatibility then show that $l$ itself is an injection.

By hypothesis $l_{0}$ is an injection. Suppose by induction that $l_{m}$ is an injection. Let $\varepsilon$ generate the kernel of $A_{m+1} \rightarrow A_{m}$. By flatness, there are identifications $\varepsilon B_{m+1} \cong(\varepsilon) \otimes_{A_{m+1}} B_{m+1} \cong B_{0}$ and $\varepsilon F_{m+1}^{n} \cong(\varepsilon) \otimes F_{m+1}^{n} \cong F_{0}^{n}$, and under these identifications, $\varepsilon \cdot l_{m+1}$ is identified with $\imath_{0}$. Now consider the diagram

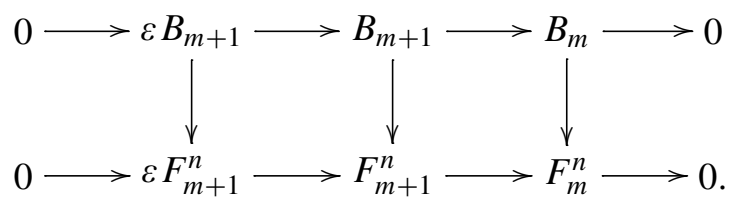

By the Snake Lemma and the inductive hypothesis, the kernel of the left-hand vertical map is identified with the kernel of the middle map (which is $\imath_{m+1}$ ). But the left-hand map is identified with $\iota_{0}$, hence is injective.

In particular, a section of $\mathscr{T}_{X / S}^{\mathcal{O}}$ over $T \rightarrow S$ lies in $\mathscr{T}_{X_{T}}^{\text {parf }}\left(X_{T}\right)$. This will be essential when we study relative generalized Azumaya algebras in Lemma 3.2.3. 
3.3. Azumaya algebras. For the sake of completeness, we recall a few basic facts about Azumaya algebras, which can be thought of as coherent models for $\mathrm{PGL}_{n^{-}}$ torsors. We suppose that $f: X \rightarrow S$ is a proper morphism of finite presentation between algebraic spaces. By abuse of notation, we will also write $f$ for the induced geometric morphism $X_{\text {rét }} \rightarrow S_{\text {ÉT }}$.

Let $G \rightarrow S$ be a flat linear algebraic $S$-group of finite presentation. It follows from the definition that $f_{*} \mathrm{~B} G_{X}$ is the stack of (étale) $G$-torsors on $X$, whose sections over an $S$-scheme $T$ are $G_{T}$-torsors on $X_{T}$.

Lemma 3.3.1. The stack $f_{*} \mathrm{~B} G_{X}$ is an Artin stack locally of finite presentation over $S$.

Sketch of proof. By the usual arguments, we may assume that $S$ is the spectrum of an excellent Noetherian ring (even a finite type $\mathbb{Z}$-algebra if we so desire) and that there is a closed immersion $G \hookrightarrow \mathrm{GL}_{n, S}$ for some $n$. It is well known that the stack $f_{*} \mathrm{BGL}_{n}$ is an Artin stack locally of finite presentation over $S$. (One can see [Laumon and Moret-Bailly 2000] for the case of $X$ projective or apply the main theorem of [Artin 1974] — the standard deformation theory of [Grothendieck 1971] and the usual Grothendieck existence theorem [1961] — in the arbitrary proper case.) Furthermore, extension of structure group yields a 1-morphism

$$
\varepsilon: f_{*} \mathrm{~B} G \rightarrow f_{*} \mathrm{BGL}_{n}
$$

it suffices to show that $\varepsilon$ is representable by algebraic spaces locally of finite presentation. To see this, let $T \rightarrow f_{*} \mathrm{BGL}_{n}$ be any morphism over $S$, corresponding to some $\mathrm{GL}_{n}$-torsor $V$ on $X_{T}$. The fiber product $f_{*} \mathrm{~B} G \times_{f_{*} \mathrm{BGL}_{n}} T$ is identified with the sheaf of reductions of structure group of $V$ to $G$, which is simply $V / G$. Thus, we will be done if we show that $f_{*}(V / G)$ is an algebraic space locally of finite presentation over $T$.

By [Artin 1974, Corollary 6.3], the quotient sheaf $V / G$ is representable by a separated algebraic space of finite presentation over $X_{T}$. The fact that $f_{*}(V / G)$ is an algebraic space may be seen in several ways. Here is one of them: we can identify it with the fiber of $\underline{\operatorname{Hom}}_{T}\left(X_{T}, V / G\right) \rightarrow \underline{\operatorname{Hom}}_{T}\left(X_{T}, X_{T}\right)$ over the section $\operatorname{id}_{X_{T}}$. Thus, it suffices to show that $\operatorname{Hom}_{T}\left(X_{T}, V / G\right)$ is an algebraic space locally of finite presentation over $T$; this is a standard result, as $X_{T}$ is proper and $V / G$ is separated. Its algebraicity follows from, for example, Artin's theorem or from the methods of [Lieblich 2006b].

In the case of $G=\mathrm{PGL}_{n, S}$, there is a natural closed immersion $G \hookrightarrow \mathrm{GL}_{n^{2}}$ given by the action of $\mathrm{PGL}_{n}$ on $\mathrm{M}_{n}(\mathrm{O})$ by conjugation (the adjoint representation). In this case, in fact, $\mathrm{PGL}_{n}=A u t_{\mathrm{alg}}\left(\mathrm{M}_{n}(\mathbb{O})\right)$. Thus, there this is a very concrete way to describe the bundles admitting a reduction of structure group to $\mathrm{PGL}_{n}$, since 
these bundles are such that the associated locally free sheaves of rank $n^{2}$ carry the structure of an Azumaya algebra.

Definition 3.3.2. An Azumaya algebra $\mathscr{A}$ of degree $n$ on a ringed topos $T$ is a form of $\mathrm{M}_{n}\left(\mathrm{O}_{T}\right)$.

More precisely, to give a reduction of structure group on a $\mathrm{GL}_{n^{2}}$-torsor is to give a multiplication on the associated locally free sheaf making it into an Azumaya algebra. The diagram

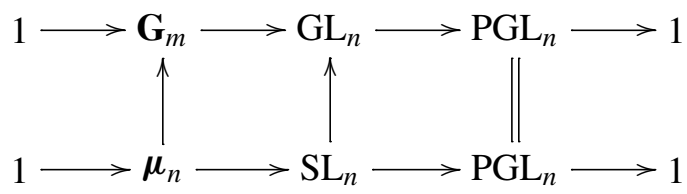

(where the horizontal sequences are exact in the fppf topology, with the bottom exact in the étale topology only if $n$ is invertible on $S$ ) gives rise to a diagram of coboundary maps in non-abelian (flat) cohomology

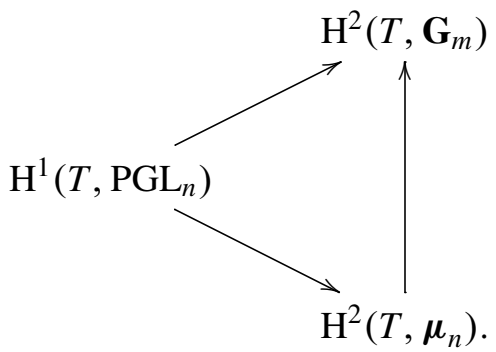

In Giraud's theory [1971, Section V.4.2], one can be more precise: given a $\mathrm{PGL}_{n^{-}}$ torsor $P \rightarrow T$, the cohomology class $\operatorname{cl}(P) \in \mathrm{H}^{2}\left(T, \mu_{n}\right)$ is precisely that given by the $\boldsymbol{\mu}_{n}$-gerbe of liftings (reductions of structure group) of $T$ to an $\mathrm{SL}_{n}$-torsor. In the language of Azumaya algebras, this is accomplished by looking at the gerbe of trivializations: a trivialization of $\mathscr{A}$ is given by a triple $(\mathscr{V}, \delta, \varphi)$ with $\mathscr{V}$ a locally free sheaf, $\delta: \operatorname{det} \mathscr{V} \stackrel{\sim}{\rightarrow} \mathcal{O}$ a trivialization of the determinant, and $\varphi: \mathscr{E}$ d $(\mathscr{V}) \stackrel{\sim}{\rightarrow} \mathscr{A}$ an isomorphism.

3.4. Twisted objects and rigidifications. In this section, we give a possible definition for a twisted object in a stack (relative to an abelian gerbe). We then review a basic stack-theoretic construction of Abramovich, Corti, and Vistoli [Abramovich et al. 2003] and show how pushing it forward naturally yields coverings by stacks of twisted objects.

3.4.1. Twisted objects. Let $\mathscr{Y} \rightarrow X$ be a stack. Suppose (for the sake of simplicity) that $A$ is an abelian sheaf on $X$ admitting a central injection $\chi: A \rightarrow \mathscr{I}(\mathscr{Y})$ into the inertia stack of $\mathscr{Y}$. Let $\mathscr{X} \rightarrow X$ be an $A$-gerbe on $X$. (Since $A$ is abelian, we 
may view this as an $X$-stack along with an identification of $A$ with the inertia stack $\Im(\mathscr{X})$.)

Definition 3.4.1.1. An $\mathscr{X}$-twisted section of $\mathscr{Y}$ over $T \rightarrow X$ is a 1-morphism $f$ : $\mathscr{L} \times{ }_{X} T \rightarrow \mathscr{Y}$ such that the induced map $A \rightarrow \mathscr{I}\left(\mathscr{X} \times{ }_{X} T\right) \rightarrow f^{*} \mathscr{I}(\mathscr{Y})$ is identified with the pullback under $f$ of the canonical inclusion $\chi: A \rightarrow \mathscr{I}(\mathscr{S})$.

The collection of $\mathscr{L}$-twisted sections of $\mathscr{Y}$ forms a substack of the Hom-stack $\underline{\operatorname{Hom}}_{X}(\mathscr{X}, \mathscr{Y})$, as the condition on the inertial morphism is local on the base of any family. We will write this substack as $\mathscr{S}^{\mathscr{L}}$. Note that there is a natural central injection $A \rightarrow \mathscr{I}\left(\mathscr{Y}^{\mathscr{X}}\right)$ given by acting on a map $\mathscr{X} \rightarrow \mathscr{Y}$ by acting on sections of $\mathscr{Y}$, or (what amounts to the same thing by the twisted condition) on the sections of $\mathscr{X}$.

The following transition results will prove useful.

Lemma 3.4.1.2. Let $\mathscr{Y}$ be an $X$-stack and $\sigma: X \rightarrow \mathscr{Y}$ a section. There is an essentially unique 1-morphism $\mathrm{B} A$ Aut $(\sigma) \rightarrow \mathscr{Y}$ sending the section corresponding to the trivial torsor to $\sigma$.

Proof. Let $\bar{\sigma} \subset \mathscr{S}$ be the stack-theoretic image of $\sigma$ (so that $\sigma$ factors as an epimorphism $X \rightarrow \bar{\sigma}$ followed by a monomorphism $\bar{\sigma} \rightarrow \mathscr{9}$ ). By definition, $\bar{\sigma}$ is the substack of $\mathscr{S}$ consisting of objects which are locally isomorphic to $\sigma(X)$. Given an object $Y$ of $\bar{\sigma}$ over some $X$-space $T$, the sheaf $\operatorname{Isom}_{T}(Y, \sigma(T))$ is an $A u t(\sigma)_{T^{-}}$ torsor; this defines a 1-morphism $\gamma: \bar{\sigma} \rightarrow \operatorname{BAdut}(\sigma)$.

To check that this is a 1 -isomorphism, we choose a cleavage for $\mathscr{Y}$. It is enough to prove that $\gamma$ is fully faithful on fiber categories, as it is clear that any torsor is locally in the image of $\gamma$. Let $Y$ and $Y^{\prime}$ be two objects of $\bar{\sigma}_{T}$, and consider the induced map of sheaves $\operatorname{Isom}\left(Y, Y^{\prime}\right) \rightarrow \operatorname{Isom}\left(\operatorname{Isom}(X, Y)\right.$, $\left.\operatorname{Isom}\left(X, Y^{\prime}\right)\right)$. Since $Y$ and $Y^{\prime}$ are both locally isomorphic to $X$, this map of sheaves is trivially a surjection. Thus, we are done once we show that it is injective, for which it suffices (by the universality of the argument) to show that it is injective on global sections.

The map described in the statement is simply the 1-inverse of $\bar{\sigma} \rightarrow \operatorname{BAdut}(\sigma)$.

Proposition 3.4.1.3. Given a section $\sigma: X \rightarrow \mathscr{X}$, the natural restriction map $\mathscr{S}^{\mathscr{X}} \rightarrow$ $\underline{\operatorname{Hom}}_{X}(X, \mathscr{Y})=\mathscr{Y}$ is a 1-isomorphism.

Proof. Given a section of $\mathscr{Y}$, the injection $A \rightarrow \mathscr{I}(\mathscr{Y})$ combined with Lemma 3.4.1.2 yields an essentially unique induced map BA $\rightarrow \mathscr{Y}$ which respects the $A$-structures on the inertia stacks. This construction gives an isomorphism $\mathscr{Y} \rightarrow \mathscr{S}^{\mathrm{B} A}$. Using $\sigma$ to identify $\mathscr{X}$ with $\mathrm{B} A$, we have just described the inverse of the natural map given in the statement.

Proposition 3.4.1.4. Let $\mathscr{X}$ and $\mathscr{Y}$ be A-gerbes on $X$. There is a natural 1-isomorphism

$$
\left(\mathscr{S}^{\mathscr{x}}\right)^{\mathscr{y}} \stackrel{\sim}{\rightarrow} \mathscr{9}^{\mathscr{y} \wedge \mathscr{X}}
$$

of stacks of twisted objects. 
Proof. Consider the diagram

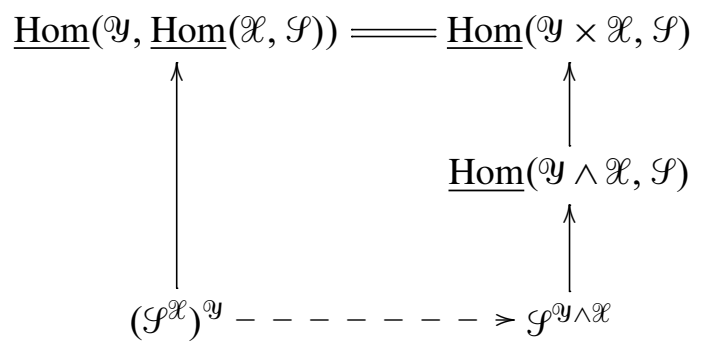

The top equality comes from the natural adjunction and the uppermost vertical right map comes from the natural map $m: \mathscr{Y} \times \mathscr{X} \rightarrow \mathscr{Y} \wedge \mathscr{X}$. The map $A \times A \rightarrow$ $\mathscr{I}(\mathscr{Y} \times \mathscr{X}) \rightarrow m^{*} \mathscr{I}(\mathscr{Y} \wedge \mathscr{X})=A$ is just the addition map, from which it follows that a unique (up to 2-isomorphism) dashed arrow exists filling in the diagram. The fact that every arrow is either an equality or an inclusion shows that the dashed arrow is a 1-isomorphism.

3.4.1.5. There is a more ad hoc description of $\mathscr{L}$-twisted objects in terms of a cocycle representing the cohomology class of $\mathscr{X}$. This can be useful for its value in constructing quick (but perhaps not philosophically satisfying) proofs, but we will not use this formalism. In order to make the definition, we must fix a cleavage (pseudo-functor structure) on $\mathscr{S}$.

Given $\mathscr{X}$, we can choose a hypercovering $U_{\bullet} \rightarrow X$ which splits $\mathscr{X}$, in the following sense:

(1) there is a section $\sigma$ of $\mathscr{X}$ over $U_{0}$, and

(2) the two pullbacks of $\sigma$ to $U_{1}$ are isomorphic, say via $\varphi$.

Computing the coboundary of $\varphi$ and using the fact that $\mathscr{X}$ is an $A$-gerbe yields a 2-cocycle $a \in \Gamma\left(U_{2}, A\right)$. It is a standard fact that this cocycle represents the same cohomology class as $\mathscr{X}$. Slightly more subtle is the fact that one can explicitly construct a gerbe from a cocycle on a hypercovering. (This gerbe is just the stack of "twisted $A$-torsors"; we will not describe it in detail here.)

Definition 3.4.1.6. Given $\left(U_{\bullet}, a\right)$ as above, a $\left(U_{\bullet}, a\right)$-twisted section of $\mathscr{Y}$ over $T \rightarrow X$ is given by

(1) a 1-morphism $\varphi: U_{0} \times{ }_{X} T \rightarrow \mathscr{Y}$, and

(2) a 2-morphism $\psi:\left(p_{0}^{1}\right)^{*} \varphi \stackrel{\sim}{\rightarrow}\left(p_{1}^{1}\right)^{*} \varphi$, where $p_{0}^{1}$ and $p_{1}^{1}$ are the two natural maps $U_{1} \times_{X} T \rightarrow U_{0} \times_{X} T$,

subject to the condition that the coboundary $\delta \psi \in \operatorname{Aut}\left(\left(p_{0}^{2}\right)^{*} \varphi\right)$ is equal to the action of $a$ (via the inclusion of $A$ in $\mathscr{(}(\mathscr{S})$ ). 
It is clear that $\left(U_{\bullet}, a\right)$-twisted objects of $\mathscr{Y}$ form a stack on $X$.

Moreover, given an $\mathscr{X}$-twisted object of $\mathscr{Y}$ over $T$, the construction of $\left(U_{\bullet}, a\right)$ induces a $\left(U_{\bullet}, a\right)$-twisted object of $\mathscr{Y}$ over $T$.

Proposition 3.4.1.7. There is a natural equivalence between the stack of $\mathscr{Q}$-twisted objects of $\mathscr{Y}$ and $\left(U_{\bullet}, a\right)$-twisted objects of $\mathscr{Y}$.

Sketch of proof. Let $f: \mathscr{X} \rightarrow \mathscr{Y}$ be an $\mathscr{L}$-twisted object of $\mathscr{Y}$. Let $\varphi: U_{0} \rightarrow \mathscr{X} \rightarrow \mathscr{Y}$ be the composition of $f$ with the map coming from the chosen trivialization of $\mathscr{L}$ over $U_{0}$. Via the cleavage on $\mathscr{Y}$, the two maps $U_{1} \rightarrow U_{0}$ give an isomorphism $\psi:\left(p_{0}^{1}\right)^{*} \varphi \stackrel{\sim}{\rightarrow}\left(p_{1}^{1}\right)^{*} \varphi$ of the pullbacks. The condition that $f$ be $\mathscr{X}$-twisted shows that the action of the coboundary is precisely multiplication by $a$, giving a $\left(U_{\bullet}, a\right)$ twisted object of $\mathscr{Y}$.

By descent theory, the statement that this gives an equivalence boils down to the proposition that a morphism $\mathscr{X} \rightarrow \mathscr{Y}$ is equivalent to a natural transformation between fibered categories. (This requires some careful justification, which can come from the realization that $\mathscr{L}$ is the stack of $a$-twisted torsors with respect to the pair $\left(U_{\bullet}, a\right)$. Since we will not use this formalism in this paper, we will not go into the rather unpleasant details.)

3.4.2. Pushing forward rigidifications. Let $\mathscr{S} \rightarrow X$ be a stack on $X$ with inertia stack $\mathscr{I}(\mathscr{Y}) \rightarrow \mathscr{Y}$. Suppose $A$ is an abelian sheaf on $X$ admitting a central injection

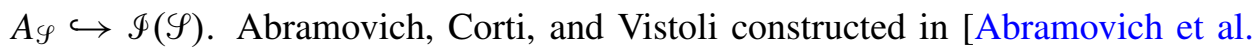
2003] the rigidification of $\mathscr{Y}$ along $A$, which we denote by $\mathscr{Y} \square \mathrm{A}$, following [Romagny 2005 , Section 5]. It is characterized by a universal property: there is a 1-morphism $\mathscr{S} \rightarrow \mathscr{S} \rrbracket$ A which is 1-universal among morphisms $\varphi: \mathscr{S} \rightarrow \mathscr{T}$ for which $A_{\mathscr{S}}$ is in the kernel of the induced map $\mathscr{I}(\mathscr{Y}) \rightarrow \varphi^{*} \mathscr{I}(\mathscr{T})$. We will freely use the standard fact that $\mathscr{Y} \rightarrow \mathscr{S} \square A$ is representable by $A$-gerbes.

Remark 3.4.2.1. While all existing references discuss rigidifications only for algebraic stacks on the category of $S$-schemes for some scheme $S$, the abstract nonsense works perfectly well for stacks on any site. We will implicitly use this in what follows.

In this section we study the morphism $f_{*} \mathscr{Y} \rightarrow f_{*}(\mathscr{Y} \square A)$. Given an $S$-space $T \rightarrow S$ and a 1-morphism $\gamma: T \rightarrow f_{*}(\mathscr{Y} \square A)$, an $A_{T}$-gerbe results on $X \times{ }_{S} T$, coming from the fact that $\mathscr{Y} \rightarrow \mathscr{Y} \square A$ is represented by $A$-gerbes and the fact that $T \rightarrow f_{*}(\mathscr{Y} \square A)$ corresponds to a morphism $X \times{ }_{S} T \rightarrow \mathscr{Y} \square A$.

Definition 3.4.2.2. With the above notation, the $A$-gerbe associated to $\gamma$ will be denoted $\mathscr{X}_{\gamma}$ and called the gerbe of $\gamma$. The class of $\mathscr{X}_{\gamma}$ in $\mathrm{H}^{2}\left(X \times_{S} T, A\right)$ will be called the (cohomology) class of $\gamma$. 
Proposition 3.4.2.3. Let $\mathscr{X} \rightarrow X$ be an A-gerbe. There is a canonical isomorphism $\mathscr{Y}^{\mathscr{X}} \square A \cong \mathscr{Y} \square$. Moreover, for any $T \rightarrow S$, a 1-morphism $\gamma: T \rightarrow f_{*}(\mathscr{Y} \square A)$ lifts to a 1-morphism $T \rightarrow f_{*}\left(\mathscr{S}^{\mathscr{X}_{\gamma}}\right)$.

Proof. Consider the diagram

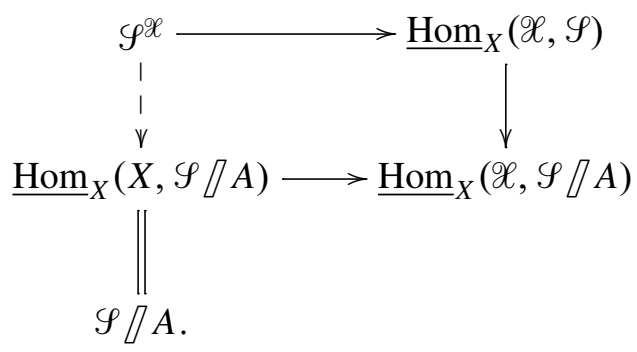

The $\mathscr{L}$-twisted condition shows that the dashed arrow exists, and since every arrow in question is a monomorphism, the dashed arrow is unique up to 2-isomorphism. By the universal property of $\rrbracket$, there results a natural morphism $v: \mathscr{Y}^{\mathscr{X}} \rrbracket A \rightarrow \mathscr{S} \rrbracket A$.

To show that $v$ is an equivalence, we may work locally on $X$ and assume that $\mathscr{X}$ is trivial. In this case, Proposition 3.4.1.3 shows that the dashed arrow in (3.4.2.3.1) is the image of a 1-isomorphism $\mathscr{S} \stackrel{\sim}{\rightarrow} \mathscr{S}^{\mathscr{X}}$ which respects the $A$-structures. It follows that the map on rigidifications is an isomorphism, as desired.

Definition 3.4.2.4. Given an $A$-gerbe $\mathscr{X} \rightarrow X$, let $f_{*}^{\mathscr{X}}(\mathscr{Y} \square A)$ denote the stacktheoretic image of $f_{*}\left(\mathscr{Y}^{\mathscr{X}}\right)$ under the natural map $f_{*}\left(\mathscr{Y}^{\mathscr{X}}\right) \rightarrow f_{*}(\mathscr{Y} \square A)$. We will call $f_{*}^{\mathscr{X}}(\mathscr{Y} \square A)$ the $\mathscr{L}$-twisted part of $f_{*}(\mathscr{Y} \square A)$.

Lemma 3.4.2.5. Given an A-gerbe $\mathscr{X} \rightarrow X$ and a 1 -morphism $\varphi: T \rightarrow f_{*}^{\mathscr{X}}(\mathscr{Y} \square A)$, there is an étale surjection $U \rightarrow T$ and an isomorphism

$$
T \times\left.\left. f_{*}^{\mathscr{P}(\mathscr{A} / A)} f_{*}\left(\mathscr{Y}^{\mathscr{X}}\right)\right|_{U} \cong f_{*} \mathrm{~B} A\right|_{U} .
$$

Proof. By construction $f_{*}\left(\mathscr{Y}^{\mathscr{X}}\right) \rightarrow f_{*}^{\mathscr{X}}(\mathscr{Y} \rrbracket A)$ is an epimorphism of stacks, so there is some $U \rightarrow T$ such that $\left.\varphi\right|_{U}$ lifts into $f_{*}\left(\mathscr{Y}^{\mathscr{X}}\right)$. Thus, it suffices to show that if $\varphi$ lifts to $f_{*}\left(\mathscr{Y}^{\mathscr{X}}\right)$ then the fiber product is isomorphic to $f_{*} \mathrm{~B} A$. In this case the gerbe $\mathscr{X}_{\gamma} \rightarrow X \times{ }_{S} T$ is isomorphic to BA. The result follows from the compatibility of the formation of fiber product with pushforward.

3.4.3. Deformation theory. In this section we assume that $f$ is a proper morphism of finite presentation between algebraic spaces and $A$ a tame constructible abelian étale sheaf.

We assume throughout this section that $f_{*} A$ and $\mathbf{R}^{1} f_{*} A$ are finite étale over $S$. (It is known that they are both constructible; if $f$ is smooth and $A$ is the pullback of a finite étale group scheme then this hypothesis will be satisfied. This will be the case in applications of interest to us.) 
Lemma 3.4.3.1. There is a natural morphism $f_{*} \mathrm{~B} A \rightarrow \mathbf{R}^{1} f_{*} A$ which realizes $f_{*} \mathrm{~B} A$ as a $f_{*} A$-gerbe over $\mathbf{R}^{1} f_{*} A$.

Proof. Recall that $\mathbf{R}^{1} f_{*} A$ is defined as the sheafification of the functor $(T \rightarrow S) \mapsto$ $\mathrm{H}^{1}\left(X \times{ }_{S} T, A\right)$ on the big étale site of $S$. A section of $f_{*} \mathrm{~B} A$ over $T \rightarrow S$ corresponds to an $A$-torsor $\mathcal{T}$ on $X \times_{S} T$. In fact, the set of isomorphism classes of objects of $f_{*} \mathrm{~B} A$ over $T$ is naturally isomorphic to $\mathrm{H}^{1}\left(X \times{ }_{S} T, A\right)$. Thus, $\mathbf{R}^{1} f_{*} A$ is the sheafification of the stack $f_{*} \mathrm{~B} A$, from which it immediately follows that $f_{*} \mathrm{~B} A \rightarrow \mathbf{R}^{1} f_{*} A$ is a gerbe. It remains to identify the inertia stack $\mathscr{I}\left(f_{*} \mathrm{~B} A\right)$. But the inertia stack of $\mathrm{B} A$ is naturally identified with $A$, from which it follows that there is a natural isomorphism $f_{*} A \stackrel{\sim}{\rightarrow} \mathscr{I}\left(f_{*} \mathrm{~B} A\right)$.

Corollary 3.4.3.2. The cotangent complex of $f_{*} \mathrm{~B} A$ over $S$ is trivial.

Proof. By the usual triangles and the fact that $\mathbf{R}^{1} f_{*} A$ is étale over $S$, it suffices to show that if $\Gamma$ is a finite étale group scheme and $\mathcal{Y} \rightarrow Y$ is a $\Gamma$-gerbe then the cotangent complex of $9 y$ over $Y$ is trivial. But this follows immediately from the fact that $\mathscr{Y}$ there is a surjection $U \rightarrow \mathscr{Y}$ such that $U$ is étale over both $\mathscr{Y}$ and $Y$.

Corollary 3.4.3.3. The natural map $\chi: f_{*}\left(\mathscr{Y}^{\mathscr{X}}\right) \rrbracket f_{*} A \rightarrow f_{*}^{\mathscr{X}}(\mathscr{Y} \rrbracket A)$ is representable by finite étale covers.

Proof. By Lemma 3.4.2.5, the fiber of $\chi$ is locally $f_{*} \mathrm{~B} A \rrbracket f_{*} A$. Applying Lemma 3.4.3.1 shows that this is precisely $\mathbf{R}^{1} f_{*} A$.

Lemma 3.4.3.4. If $f: \mathscr{S} \rightarrow \mathscr{S}^{\prime}$ is a map of $S$-stacks which is representable by fppf morphisms of algebraic stacks then $\mathscr{Y}$ is algebraic if and only if $\mathscr{Y}^{\prime}$ is.

Proof. First, we show that the diagonal of $\mathscr{S}$ is separated, quasicompact, and representable by algebraic spaces if and only if the same is true for $\mathscr{S}^{\prime}$. To this end, let $T^{\prime} \rightarrow \mathscr{Y}^{\prime} \times \mathscr{S}^{\prime}$ be a morphism with $T^{\prime}$ an affine scheme. Consider the diagram

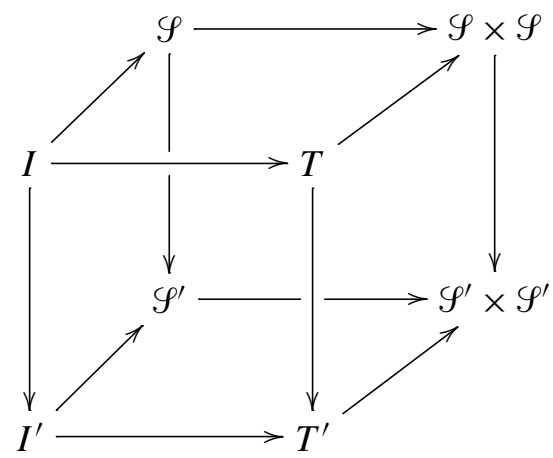

whose terms we now explain. The sheaf $I^{\prime}$ is the pullback of $T^{\prime}$ along the diagonal. By assumption, the fiber product $\mathscr{Y} \times \mathscr{S} \times \mathscr{S}^{\prime} \times \mathscr{Y}^{\prime} T^{\prime}$ is an algebraic stack over $T^{\prime}$ with fppf structure morphism. Thus, we may let $T$ be a scheme which gives a smooth 
cover, and then we let $I$ be the pullback sheaf of $T$ along the diagonal of $\mathscr{Y}$. We see that $I \rightarrow I^{\prime}$ is relatively representable by fppf morphisms of algebraic spaces. By a result of Artin [Laumon and Moret-Bailly 2000, 10.1], I is an algebraic space if and only if $I^{\prime}$ is.

It remains to show that $\mathscr{Y}$ has a smooth cover by an algebraic space if and only if $\mathscr{Y}^{\prime}$ does. In fact, it suffices to replace the word "smooth" by "fppf," by Artin's theorem [ibid.]. But then the statement is clear.

Proposition 3.4.3.5. Given an $X$-stack $\mathscr{S}$ and an A-gerbe $\mathscr{X} \rightarrow X$, the stack $f_{*}\left(\mathscr{Y}^{\mathscr{X}}\right)$ is an Artin (resp. DM) stack if and only if the stack $f_{*}^{\mathscr{X}}(\mathscr{Y} \square A)$ is an Artin (resp. DM) stack.

Proof. This follows immediately from Lemmas 3.4.2.5, 3.4.3.1, and 3.4.3.4.

3.4.3.6. We apply the preceding considerations to give a relation between certain virtual fundamental classes on $f_{*}\left(\mathscr{Y}^{\mathscr{X}}\right)$ and $f_{*}^{\mathscr{X}}(\mathscr{Y} \square A)$.

Proposition 3.4.3.7. Let $\xi: \mathscr{L} \rightarrow \mathscr{W}$ be a map of $S$-stacks. Suppose there is a tame finite étale group scheme $G \rightarrow S$ and a central injection $G_{\mathscr{L}} \hookrightarrow \mathscr{I}(\mathscr{L})$ such that

(1) the map $G_{\mathscr{L}} \rightarrow \mathscr{I}(\mathscr{L}) \rightarrow \xi^{*} \mathscr{I}(\mathscr{W})$ is trivial;

(2) the induced 1-morphism $\mathscr{L} \rrbracket G \rightarrow \mathscr{W}$ is representable by finite étale morphisms of degree invertible on $W$.

Given a perfect complex $\mathscr{E} \in \mathbf{D}(\mathcal{W})$ and a map $\xi^{* \mathscr{E}} \rightarrow L_{\mathscr{L}} / S$ which gives a perfect obstruction theory, there is a map $\mathscr{E} \rightarrow L_{\mathscr{W} / S}$ giving a perfect obstruction theory.

Proof. Write $\overline{\mathscr{L}}:=\mathscr{E} \rrbracket G$. We have a diagram $\mathscr{L} \rightarrow \overline{\mathscr{L}} \rightarrow \mathscr{W}$ with the property that the relative cotangent complex of any pair vanishes. We first claim that any map $\xi^{* \mathscr{E}} \rightarrow L_{\mathscr{L} / S}$ is the pullback of a map $\left.\mathscr{E}\right|_{\overline{\mathscr{L}}} \rightarrow L_{\overline{\mathscr{L}} / S}$. This follows from the fact that $A$ acts trivially on the sheaves making up the complexes $\mathscr{E}$ and $L_{\mathscr{L}} / S$ and the usual description of sheaves on gerbes in terms of the representation theory of $A$.

Thus, to prove the result, we are reduced to the case where $\mathscr{L} \rightarrow \mathscr{W}$ is representable by finite étale maps with invertible degrees. In this case, there is a splitting trace map given by dividing the trace of the covering by the degree. Note that $L_{\mathscr{q} / S}=\xi^{*} L_{\mathscr{W} / S}$, so that the perfect obstruction theory becomes a map $\alpha: \xi^{* \mathscr{E}} \rightarrow \xi^{*} L_{\mathscr{W} / S}$. Taking the splitting trace produces a map $\mathscr{E} \rightarrow L_{\mathscr{W} / S}$, which is a perfect obstruction theory because it is a summand of $\xi_{*} \alpha$.

Corollary 3.4.3.8. A complex in $\mathbf{D}\left(f_{*}^{\mathscr{X}}\left(\mathscr{Y}_{\square} \not A\right)\right)$ can be realized as a perfect obstruction theory if and only if its pullback to $f_{*}\left(\varphi^{\mathscr{D}}\right)$ can be realized as a perfect obstruction theory. 


\section{Compactified moduli of $\mathrm{PGL}_{\boldsymbol{n}}$-torsors: an abstract approach}

In this section we compactify the moduli of $\mathrm{PGL}_{n}$-torsors using the techniques of Section 3.4 and use the structure of our compactification to prove the Irreducibility Theorem. In Section 5 we will give a more concrete description of the abstract compactification we construct here and use it to describe the virtual fundamental class on the moduli stack.

Throughout this section, $f: X \rightarrow S$ will be a proper flat morphism of finite presentation between algebraic spaces, $n$ will be an integer invertible on $S$, and $\pi: \mathscr{X} \rightarrow X$ will be a fixed $\mu_{n}$-gerbe. We will abuse notation and let $f$ stand for the geometric morphism $X_{\text {rét }} \rightarrow S_{\text {ÉT }}$ as well.

4.1. Twisted sheaves. We briefly describe how the theory developed in Section 3.4.1 works out in the case of twisted objects of the stack of coherent sheaves on $X / S$. We fix the $\mu_{n}$-gerbe $\mathscr{X} \rightarrow X$ and do the twisting with respect to the natural inclusion of $\boldsymbol{\mu}_{n}$ into the inertia stack of $f_{*} \mathscr{C}_{X / S}$ (see Proposition 3.2.2).

Definition 4.1.1. An $\mathscr{L}$-twisted object of $f_{*} \mathscr{C}_{X / S}$ over $T \rightarrow S$ is called a flat family of $\mathscr{L}$-twisted coherent sheaves parametrized by $T$.

If the fibers of the family are torsion-free, we will speak of a flat family of torsion-free $\mathscr{L}$-twisted sheaves, etc. The reader is referred to [Lieblich 2007, paragraph 2.2.6.3] for a discussion of associated points, purity, and torsion-free sheaves on Artin stacks.

Concretely, an $\mathscr{X}$-twisted sheaf is a sheaf $\mathscr{F}$ on $\mathscr{X}$ such that the representation of $\boldsymbol{\mu}_{n}$ on each geometric fiber of $\mathscr{F}$ is given by scalar multiplication. These sheaves were originally introduced in [Giraud 1971] and have found various recent applications in mathematical physics and in algebra.

Notation 4.1.2. The stack of totally pure $\mathscr{L}$-twisted coherent sheaves with rank $n$ and trivialized determinant will be denoted $\mathbf{T w}_{\mathscr{L} / S}(n, \mathcal{O})$.

It is relatively straightforward to prove that $\mathbf{T} \mathbf{w}_{\mathscr{L} / S}(n, \mathcal{O})$ is an Artin stack locally of finite presentation over $S$. This is done in detail in section 2.3 of [Lieblich 2007]. Earlier work on twisted sheaves in the context of elliptic fibrations and $K 3$ surfaces was carried out by Căldăraru [2002a; 2002b], and a study of their moduli for projective varieties, with a description of the moduli spaces associated to $K 3$ and abelian surfaces, by [Yoshioka 2006]. Applications of Yoshioka's results to a conjecture of Căldăraru were discovered by Huybrechts and Stellari (described in the appendix of [Yoshioka 2006]). The abstract approach taken here has also proven useful in the study of certain arithmetic questions [Lieblich 2008b]. When $\mathscr{X}$ admits a locally free twisted sheaf $\mathscr{V}$ (that is, when its class in $\mathrm{H}^{2}\left(X, \mathbf{G}_{m}\right)$ lies in the Brauer group of $X), \mathscr{L}$-twisted sheaves are equivalent to $\pi_{*} \mathscr{E}[\mathbf{E} d(\mathscr{V})$-modules, where the problem of constructing moduli under various stability conditions was 
first studied in [Simpson 1994]. The case in which the $\pi_{*} \mathscr{E}(\mathfrak{d}(\mathscr{V})$-modules have rank 1 was studied in [Hoffmann and Stuhler 2005]; they also produced a symplectic structure on the moduli space when $X$ is a $K 3$ or abelian surface, giving results analogous to those of Yoshioka.

4.2. Compactification by rigidification. The natural map $\mathrm{SL}_{n} \rightarrow \mathrm{PGL}_{n}$ gives an "extension of structure group" morphism $\varepsilon: \mathrm{BSL}_{n} \rightarrow \mathrm{BPGL}_{n}$.

Lemma 4.2.1. The map $\varepsilon$ induces an isomorphism $\mathrm{BSL}_{n} \rrbracket \boldsymbol{\mu}_{n} \stackrel{\sim}{\rightarrow} \mathrm{BPGL}_{n}$.

Proof. Given a stack $\mathscr{Y}$, there is a natural equivalence of categories between morphisms $\mathrm{BSL}_{n} \rightarrow \mathscr{Y}$ and $\mathrm{SL}_{n}$-equivariant objects of $\mathscr{Y}$. (The reader is referred to [Kovács and Lieblich 2006, Section 3.A] for a description of this equivalence.) On the other hand, there is clearly a natural equivalence between $\mathrm{PGL}_{n}$-equivariant objects of $\mathscr{S}$ and $\mathrm{SL}_{n}$-equivariant objects on which the $\mu_{n} \subset \mathrm{SL}_{n}$ acts trivially. But these correspond precisely to morphisms $\mathrm{BSL}_{n} \rightarrow \mathscr{S}$ such that the induced map on inertia annihilates $\mu_{n} \subset \mathscr{I}\left(\mathrm{BSL}_{n}\right)$. The lemma follows from the universal property of the rigidification.

Taking the associated locally free sheaf with trivialized determinant yields an inclusion $\mathrm{BSL}_{n} \subset \mathscr{T}_{X / S}^{\mathbb{O}}(n)$. (Note that the natural target is not $\mathscr{P}_{X / S}^{\mathbb{O}}(n)$ unless the fibers of $X / S$ are Cohen-Macaulay.) Moreover, there is a natural inclusion $\boldsymbol{\mu}_{n} \hookrightarrow \mathscr{I}\left(\mathscr{T}_{X / S}^{O}(n)\right)$ extending the inclusion over $\mathrm{BSL}_{n}$. It follows that there is an inclusion $\mathrm{BPGL}_{n} \hookrightarrow\left(\mathscr{T}_{X / S}^{\mathrm{O}}(n) \square \boldsymbol{\mu}_{n}\right)$.

There is a natural morphism from $\chi: f_{*} \mathrm{BPGL}_{n} \rightarrow \mathbf{R}^{2} f_{*} \boldsymbol{\mu}_{n}$ which we may define as follows. (Note that since $X$ is proper over $S$, the sheaf $\mathbf{R}^{2} f_{*} \boldsymbol{\mu}_{n}$ on $S_{\text {ÉT }}$ is a quasifinite algebraic space of finite presentation by Artin's theorem. A proof in terms of algebraic spaces may be found in the last chapter of [Artin 1973].) Given an object of $f_{*} \mathrm{BPGL}_{n}=f_{*}\left(\mathrm{BSL}_{n} \rrbracket \boldsymbol{\mu}_{n}\right)$ over some $T \rightarrow S$, there is an associated $\boldsymbol{\mu}_{n}$-gerbe on $X \times{ }_{S} T$ (see Definition 3.4.2.2), and we simply take the image in $\mathrm{H}^{0}\left(T, \mathbf{R}^{2} f_{*} \boldsymbol{\mu}_{n}\right)$.

Lemma 4.2.2. The stack $f_{*}\left(\mathscr{T}_{X / S}^{O}(n) \square \boldsymbol{\mu}_{n}\right)$ is an Artin stack locally of finite presentation over $S$. If in addition $f$ is smooth then the stack is quasiproper.

Proof. Since $\mathbf{R}^{2} f_{*} \boldsymbol{\mu}_{n}$ is an algebraic space, it suffices to show that $\chi$ makes $f_{*}\left(\mathscr{T}_{X / S}^{\mathcal{O}}(n)\right)$ into an algebraic $\left(\mathbf{R}^{2} f_{*} \boldsymbol{\mu}_{n}\right)$-stack of finite presentation. To prove this, it suffices to work locally on $\mathbf{R}^{2} f_{*} \boldsymbol{\mu}_{n}$. Thus, as any section of $\mathbf{R}^{2} f_{*} \boldsymbol{\mu}_{n}$ (and in particular, the "universal section" given by the identity map) is locally associated to the cohomology class of a $\boldsymbol{\mu}_{n}$-gerbe, we see that it suffices to prove that, given a $\boldsymbol{\mu}_{n}$-gerbe $\mathscr{X} \rightarrow X$, the stack $f_{*}^{\mathscr{X}}\left(\mathscr{T}_{X / T}^{\mathcal{O}}(n) \rrbracket \boldsymbol{\mu}_{n}\right)$ is an Artin stack locally of finite presentation. Applying Proposition 3.4.3.5, we see that it suffices to prove that $f_{*}\left(\left(\mathscr{T}_{X / T}^{\mathbb{O}}(n)\right)^{\mathscr{X}}\right)$ is an Artin stack locally of finite presentation. But this is an open 
substack of the stack of perfect $\mathscr{L}$-twisted sheaves with trivialized determinant, which is known to be Artin and locally of finite presentation. (For the proof that it is an Artin stack, the reader is referred to [Lieblich 2007, Section 2.3]. The condition that the fibers be perfect is clearly an open condition, and closed if $X / S$ is smooth.)

Suppose $f$ is smooth, and let $(R,(t), \kappa)$ be a discrete valuation ring over $S$ with fraction field $K$. Suppose Spec $K \rightarrow f_{*}\left(\mathscr{T}_{X / S}^{\mathscr{O}}(n) \square \boldsymbol{\mu}_{n}\right)$ is a 1-morphism. We may suppose without loss of generality that $S=\operatorname{Spec} R$. Since $f$ is proper and smooth, $\mathbf{R}^{2} f_{*} \boldsymbol{\mu}_{n}$ is finite and étale over $R$. Making a finite base change (which is permitted in the stacky version of the valuative criterion), we may assume that $\mathbf{R}^{2} f_{*} \boldsymbol{\mu}_{n}$ is a disjoint union of sections over Spec $R$. It follows that to prove that $f_{*}\left(\mathscr{T}^{O}(n) / \boldsymbol{\mu}_{n}\right)$ is quasiproper, it suffices to prove that $f_{*}\left(\left(\mathscr{T}_{X / S}^{\mathscr{O}}(n)\right)^{\mathscr{L}}\right)$ is quasiproper, where $\mathscr{X} \rightarrow X$ is an arbitrary $\boldsymbol{\mu}_{n}$-gerbe. Since $X$ is regular, the condition that the twisted sheaf be perfect is trivial, and the result comes down to showing that given a discrete valuation ring $R$ and a torsion-free $\mathscr{L}$-twisted sheaf $\mathscr{F}$ of rank $n$ with trivialized determinant over the generic point of $R$, there is an extension of $\mathscr{F}_{F}$ to a flat family over a finite flat extension of $R$ such that the trivialization of the determinant extends.

Let $K$ be the fraction field of $R$ and $\kappa$ its residue field. It is easy to see that any flat extension $\mathscr{G}$ of $\mathscr{F}$ will have trivial determinant (as all invertible sheaves on $\operatorname{Spec} R$ are trivial). Choose an isomorphism $l: \operatorname{det} \mathscr{G} \stackrel{\sim}{\rightarrow}$ O. Composing with the fixed generic isomorphism det $\mathscr{F} \stackrel{\sim}{\rightarrow} \mathcal{O}_{X_{K}}$ yields an injection $\alpha: \operatorname{det} \varphi \rightarrow \mathcal{O}_{X_{K}}$ (the latter being viewed as a sheaf on $X$ by pushforward from $X_{K}$ ). Since $X$ is geometrically connected, the trivial invertible $O_{X_{R}}$-subsheaves of $O_{X_{K}}$ all have the form $t^{s} O_{X_{R}}$ for some $s \in \mathbb{Z}$. Taking an $n$-th root of $t$ if necessary (which may result in a finite extension of $R$ ), we may assume that $s=n s^{\prime}$ for some integer $s^{\prime}$. Replacing $\mathscr{G}$ by $\mathscr{G}\left(t^{-s^{\prime}}\right)$ yields $\operatorname{det} \mathscr{G}\left(t^{-s^{\prime}}\right)=(\operatorname{det} \mathscr{G})\left(t^{-s}\right)$. Thus, via $l$ and the given isomorphism $\operatorname{det} \mathscr{F} \stackrel{\sim}{\rightarrow} \mathcal{O}$, $\operatorname{det} \mathscr{G}\left(t^{-s^{\prime}}\right)$ gets identified with $t^{-s} t^{s} \mathcal{O}$, that is, $\imath$ yields a trivialization of $\operatorname{det} \mathscr{G}\left(t^{-s^{\prime}}\right)$ which extends that of $\operatorname{det} \mathscr{F}$, as desired.

Lemma 4.2.3. The natural map $f_{*} \mathrm{BPGL}_{n} \rightarrow f_{*}\left(\mathscr{T}_{X / S}^{\mathbb{O}}(n) \square \boldsymbol{\mu}_{n}\right)$ is representable by open immersions.

Proof. It again suffices to prove this for $f_{*} \operatorname{BSL}_{n}^{\mathscr{L}}$ and $f_{*}\left(\mathcal{T}_{X / S}^{\mathscr{O}}(n)\right)^{\mathscr{L}}$, where we note that $f_{*} \mathrm{BSL}_{n}^{\mathscr{X}}$ parametrizes locally free $\mathscr{L}$-twisted sheaves of rank $n$ and trivialized determinant and hence constitutes an open substack, as desired.

4.2.4. When the fibers of $X / S$ are Cohen-Macaulay, the entire discussion from the beginning of the section until the present paragraph also yields a compactification coming from the induced inclusion $\mathrm{BPGL}_{n} \hookrightarrow \mathscr{P}_{X / S}^{\mathbb{O}}$. We omit the details; the statements of the results are literally identical, with $\mathscr{P}$ replacing $\mathscr{T}$. Since $\mathscr{T}_{X / S}^{\mathscr{O}}$ is much larger than $\mathscr{P}_{X / S}^{\mathbb{O}}$, it is preferable to use the latter whenever possible. Thus, 
for example, if $X / S$ is a smooth morphism, then there results an open immersion into a quasiproper Artin stack $f_{*} \mathrm{BPGL}_{n} \hookrightarrow f_{*}\left(\mathscr{P}_{X / S}^{\mathbb{O}}(n) / / \boldsymbol{\mu}_{n}\right)$. This latter stack will play an important role in what follows. We endow it with the following notation.

Notation 4.2.5. Given a $\boldsymbol{\mu}_{n}$-gerbe $\mathscr{X}$, let $\mu_{n}^{\mathscr{X}}:=f_{*}^{\mathscr{X}}\left(\mathscr{P}_{X / S}^{\mathbb{O}} \| \boldsymbol{\mu}_{n}\right)$.

There is a surjective map $\mathbf{T w}_{\mathscr{L} / S}(n, \mathcal{O}) \rightarrow \mathcal{M}_{n}^{\mathscr{X}}$ which is universally closed and submersive.

\section{An explicit description of $M_{n}^{\mathscr{L}}$ : generalized Azumaya algebras}

In this section, we use certain algebra objects of the derived category to give a concrete description of $\mathcal{M}_{n}^{\mathscr{X}}$. Using this description, we will show that when $X$ is a smooth projective surface and $\mathscr{X} \rightarrow X$ has order $n$ in $\mathrm{H}^{2}\left(X, \mathbf{G}_{m}\right)$ then $M_{n}^{\mathscr{X}}$ has a virtual fundamental class.

5.1. Derived Skolem-Noether. In this section, we work primarily in the derived category of modules over a local commutative ring $(\mathfrak{O}, \mathfrak{m}, k)$. For the sake of a smoother exposition, we assume that $\mathcal{O}$ is Noetherian, but this is unnecessary. On occasion, we will work in the category of chain complexes. However, we will use the word "complex" in both settings; it will be clear in context whether we mean an object of $\mathbf{D}(0)$ or an object of K(O). Similarly, "isomorphism" will be consistently used in place of "quasiisomorphism" and we will always assume that isomorphisms preserve whatever additional structures of objects are implicit. Given a scheme $X$, the symbol $\mathbf{D}(X)$ will denote a derived category of sheaves of $O_{X}$-modules, with various conditions (boundedness, perfection, quasicoherence of cohomology) clear from context. In the end, it will suffice to work in the category denoted $\mathbf{D}_{\mathrm{fTd}}(X)$ in [Hartshorne 1966], so the hypotheses on $\mathbf{D}$ will not be a focus of attention.

Definition 5.1.1. Given a scheme $X$, an object $A \in \mathbf{D}(X)$ will be called a weak O-algebra if there are maps $\mu: A \stackrel{\mathbf{L}}{\otimes} \rightarrow A$ and $i: \mathcal{O} \rightarrow A$ in $\mathbf{D}(X)$ which satisfy the usual axioms for an associative unital algebra, the diagrams being required to commute in the derived category.

In other words, a weak algebra is an algebra object of the derived category. Note that the derived tensor product makes $\mathbf{D}(X)$ into a symmetric monoidal additive category (as the universal property of derived functors ensures that all different associations of an iterated tensor product are naturally isomorphic). Thus, it makes sense to speak of "associative" algebra structures.

Given an additive symmetric monoidal category, one can define most of the usual objects and maps of the theory of algebras: (unital) modules, bimodules, linear maps, derivations, inner derivations, maps of algebras, etc. We leave it to the reader to write down precise definitions of these terms, giving two examples: 
Given a map of weak algebras $A \rightarrow B$, an 0 -linear derivation from $A$ to $B$ is a map $\delta: A \rightarrow B$ in $\mathbf{D}(X)$ such that

$$
\delta \circ \mu_{A}=\mu_{B} \circ(\text { id } \stackrel{\mathbf{L}}{\otimes} \delta+\delta \stackrel{\mathbf{L}}{\otimes} \text { id })
$$

in $\mathbf{D}(X)$. A derivation from $A$ to $A$ is inner if $\delta=\mu \circ(\alpha \stackrel{\mathbf{L}}{\otimes}$ id $)-\mu \circ($ id $\stackrel{\mathbf{L}}{\otimes} \alpha)$ for some $\alpha: 0 \rightarrow A$.

Given a ring map $\mathbb{O} \rightarrow \mathcal{O}^{\prime}$, the derived functor $(\cdot) \stackrel{\mathbf{L}}{\otimes} \mathcal{O}^{\prime}: \mathbf{D}(\mathcal{O}) \rightarrow \mathbf{D}\left(\mathcal{O}^{\prime}\right)$ respects the monoidal structure. There results a natural base change operation for weak algebras and modules. (This operation will be consistently written as a change of base on the right to avoid sign errors.)

Similarly, given a weak algebra $A$ and a left $A$-module $P$, the functor $P \stackrel{\mathbf{L}}{\otimes}(\cdot)$ takes objects of $\mathbf{D}(O)$ to $A$-modules. This follows from the natural associativity of the derived tensor product.

The first nontrivial example of a weak algebra is given by

$$
\mathbf{R E n d}(K):=\mathbf{R H o m}(K, K)
$$

for a perfect complex $K$. Replacing $K$ by a projective resolution, one easily deduces the weak algebra structure from the usual composition of functions: if we write $K$ as a finite complex of free modules (which we will also call $K$ ), then $\operatorname{REnd}(K)$ has as its $n$-th module $\prod_{p} \operatorname{Hom}\left(K^{p}, K^{p+n}\right)$, with differential

$$
\partial^{n}\left(\alpha_{p}\right)_{q}=(-1)^{n+1} \alpha_{q+1} d+d \alpha_{q} .
$$

Since $K$ is perfect, the $n$-th module of $\operatorname{REnd}(K) \stackrel{\mathbf{L}}{\otimes} \mathbf{R E n d}(K)$ is equal to

$$
\prod_{a+b=n} \prod_{s, t} \operatorname{Hom}\left(K^{s}, K^{s+a}\right) \otimes \operatorname{Hom}\left(K^{t}, K^{t+b}\right)
$$

and the multiplication projects to the factors with $s=t+b$ and then composes functions as usual. Setting $K^{\vee}=\operatorname{RHom}(K, \mathcal{O})$ (the derived dual of $K$ ), we have the following basic lemma.

Lemma 5.1.2. Let $K$ be a perfect complex.

(i) There is a natural isomorphism $K \underset{\otimes}{\otimes} K^{\vee} \stackrel{\sim}{\rightarrow} \mathbf{R E n d}(K)$.

(ii) There is a natural left action of $\mathbf{R E n d}(K)$ on $K$.

Tensoring the action $\mathbf{R E n d}(K) \stackrel{\mathbf{L}}{\otimes} K \rightarrow K$ with $K^{\vee}$ on the right and using (i) yields the multiplication of $\mathbf{R E n d}(K)$.

It is essential that the action be written on the left (when using the standard sign convention for forming the total complex of a double complex [Illusie 1971, I.1.2.1], [Matsumura 1989, Appendix]) and that $K^{\vee}$ be written on the right for the signs to work out. These kinds of sign sensitivities abound in the derived category and require vigilance. 
An algebra of the form $\mathbf{R E n d}(K)$ will be called a derived endomorphism algebra. Our goal is to reprove the classical results about matrix algebras for derived endomorphism algebras of perfect complexes.

Notation 5.1.3. The symbols $P$ and $Q$ will always be taken to mean perfect complexes with a chosen realization as a bounded complex of finite free modules. Thus, maps $P \rightarrow Q$ in the derived category will always come from maps of the "underlying complexes" (taken to mean the chosen realizations). Similarly, REnd $(P)$ will have as chosen representative the complex constructed from the underlying complex of $P$ as above: $\operatorname{REnd}(P)^{n}=\prod_{t} \operatorname{Hom}\left(P^{t}, P^{t+n}\right)$ with differential $\partial\left(\alpha_{t}\right)_{s}=(-1)^{n+1} \alpha_{s+1} d+d \alpha_{s}$.

These conventions facilitate making certain basic arguments without speaking of replacing the object by a projective resolution, etc., but it is ultimately only important for this book-keeping reason; the reader may ignore it without fear (until it is explicitly invoked!).

Definition 5.1.4. Given $M \in \mathbf{D}(\mathcal{O})$, the annihilator of $M$ is the kernel $\operatorname{Ann}(M)$ of the natural map from $\mathcal{O}$ to $\operatorname{End}_{\mathbf{D}(\mathcal{O})}(M)$. The quotient $\mathcal{O} / \operatorname{Ann}(M)$ will be denoted by $\mathrm{O}_{M}$.

Given an isomorphism $\psi: P \rightarrow Q(n)$, there is an isomorphism

$$
\psi^{*}: \operatorname{REnd}(P) \rightarrow \operatorname{REnd}(Q)
$$

given by functorial conjugation by $\psi$ followed by the natural identification of $\operatorname{REnd}(Q(n))$ with $\operatorname{REnd}(Q)$. We will call this the induced map. The map $\psi^{*}$ may also be described as follows: under the natural identification of $\operatorname{REnd}(P)$ with $P \stackrel{\mathbf{L}}{\otimes} P^{\vee}, \psi^{*}$ is identified with $\psi \stackrel{\mathbf{L}}{\otimes}\left(\psi^{\vee}\right)^{-1}$.

Theorem 5.1.5. Let $P$ and $Q$ be nonzero perfect complexes of 0 -modules. If $\mathbf{R E n d}(P) \cong \mathbf{R E n d}(Q)$ as weak algebras, then there exists a unique $n$ such that the map

$$
\operatorname{Isom}(P, Q(n)) \rightarrow \operatorname{Isom}(\operatorname{REnd}(P), \operatorname{REnd}(Q))
$$

is surjective with each fiber a torsor under $O_{P}^{\times}$. If $P=Q$, then $n=0$ and the kernel is naturally a split torsor.

Corollary 5.1.6. The sequence

$$
0 \rightarrow \mathrm{O}_{P} \rightarrow \operatorname{End}(P) \rightarrow \operatorname{Der}(\operatorname{REnd}(P)) \rightarrow 0
$$

is exact. More generally, if $P$ and $Q(n)$ are isomorphic, then the map

$$
\operatorname{Hom}(P, Q(n)) \rightarrow \operatorname{Der}(\operatorname{REnd}(P), \operatorname{REnd}(Q))
$$

is surjective with each fiber naturally a torsor under $\mathrm{O}_{P}$. 
Proof. Apply Theorem 5.1.5 to $P[\varepsilon]$ (as a complex over $O[\varepsilon]$ ) and look at automorphisms of the weak algebra $\operatorname{REnd}_{\mathscr{C}[\varepsilon]}(P[\varepsilon])$ reducing to the identity modulo $\varepsilon$.

The proof of Theorem 5.1.5 will make use of the completion of 0 to lift the classical theorems on matrix algebras from the closed fiber by "infinitesimal induction."

\section{Proposition 5.1.7. If $\mathcal{O}$ is a field $k$ then Theorem 5.1.5 and Corollary 5.1.6 hold.}

Proof. The bounded derived category of $k$ is naturally identified with the category of $\mathbb{Z}$-graded finite $k$-modules by sending a complex to the direct sum of its cohomology spaces. Given perfect complexes $P$ and $Q$, the algebras $\operatorname{REnd}(P)$ and $\operatorname{REnd}(Q)$ are then each identified with a matrix algebra carrying the induced grading from the grading of the vector spaces $P$ and $Q$, respectively, and an isomorphism from $\mathbf{R E n d}(P) \rightarrow \mathbf{R E n d}(Q)$ is identified with an isomorphism of matrix algebras which respects the gradings. By the allowance of a shift, we may restrict our attention to graded spaces whose minimal nonzero graded piece is in degree 0 ; any reference in what follows to graded vector spaces will implicitly assume this hypothesis. (Of course the algebras involved will still carry nontrivial graded pieces with negative degrees.)

Let $A$ be a graded matrix algebra of rank $n^{2}$ and $V$ and $W$ two graded $n$ dimensional vector spaces with nontrivial graded $A$-actions. From the SkolemNoether theorem, we see that there is an $A$-linear isomorphism $\alpha: V \rightarrow W$. We claim that $\alpha$ is graded. To prove this, it suffices to show that given a nonzero vector $v \in V_{0}, \alpha(v)$ is in $W_{0}$ (because $V$ and $W$ are simple $A$-modules). Write $\alpha(v)=\sum w_{i}$. Since $V$ is a simple $A$ - module, $A_{n} \cdot v=V_{n}$; a similar statement holds for $W$ (given a choice of nonzero weight 0 vector, which exists by the hypothesis on the gradings). Thus, the highest nontrivial grading $N$ of $A$ will equal the highest nontrivial grading of both $V$ and $W$. Furthermore, given any $i$ such that $w_{i} \neq 0$, the fact that $A_{-i} \cdot w_{i}=W_{0}$ means that $A_{-i} \neq 0$. Given $i>0$ such that $w_{i} \neq 0$, we have for all $\tau \in A_{-i}$ that

$$
0=\alpha(0)=\alpha(\tau(v))=\tau(\alpha(v))=\tau\left(\sum w_{j}\right)=\tau\left(w_{i}\right)+\text { higher terms. }
$$

Thus, $\tau\left(w_{i}\right)=0$, which implies that $W_{0}=0$. This contradicts the assertion that $W_{0}$ is the minimal nontrivial graded piece. So $w_{i}=0$ for all $i>0$ and therefore $w \in W_{0}$. Translating this back into the derived language, we have proven that given an isomorphism $\varphi: \operatorname{REnd}(P) \rightarrow \operatorname{REnd}(Q)$, there is an isomorphism $P \rightarrow Q$ in $\mathbf{D}(k)$ which induces $\varphi$ by functoriality. In fact, we have shown the rest of the proposition as well, because $\alpha$ is the unique choice for such an isomorphism up to scalars by the Skolem-Noether theorem.

To prove Corollary 5.1.6, let $V=\oplus V_{i}$ be a graded vector space and $T \in \operatorname{End}(V)$ a noncentral linear transformation. We wish to show that if the (nontrivial) inner 
derivation by $T$ is homogeneous of degree 0 then $T$ is homogeneous of degree 0 . To do this, consider the restriction of $T$ to the degree 0 part of $\operatorname{End}(V)$. Let $T^{n}$ be a graded component of $T$ (so that $T^{n}: V \rightarrow V$ shifts degrees by $n$ ). Let $V^{m}$ be a graded component such that the induced transformation $T^{n}: V^{m} \rightarrow V^{m+n}$ is nonzero. Consider the graded linear transformation (of degree 0) $S: V \rightarrow V$ which acts as the identity on $V^{m}$ and annihilates every other component. It is easy to see that the commutator $\left[T^{n}, S\right]$ is $T^{n} S$, which implies that $[T, S]$ has a nontrivial component of degree $n$. Since the derivation $[T,-]$ is homogeneous of degree 0 , it follows that $n=0$, and thus $T$ is homogeneous of degree 0 , as desired.

Lemma 5.1.8. Theorem 5.1.5 is true for $\mathbb{O}$ if it is true for $\widehat{0}$.

Proof. We proceed by reducing the problem to a question of linear algebra and then using the faithful flatness of completion.

Suppose given $P$ and $Q$ and an isomorphism $\varphi: \operatorname{REnd}(P) \rightarrow \operatorname{REnd}(Q)$; this defines an action of $A:=\mathbf{R} \operatorname{End}(P)$ on $Q$. We claim that finding $u: P \rightarrow Q$ such that $\varphi=u^{*}$ is equivalent to finding an $A$-linear isomorphism from $P$ to $Q$. Indeed, suppose $u: P \rightarrow Q$ is $A$-linear, so that the diagram

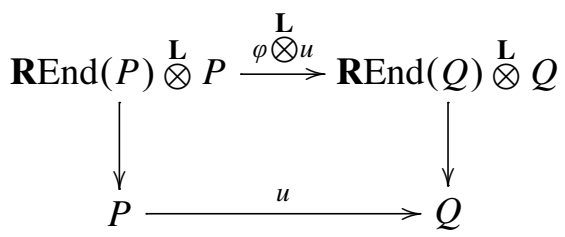

commutes, where the vertical arrows are the actions. Tensoring the left side with $P^{\vee}$ and the right side with $Q^{\vee}$, we see that the resulting diagram

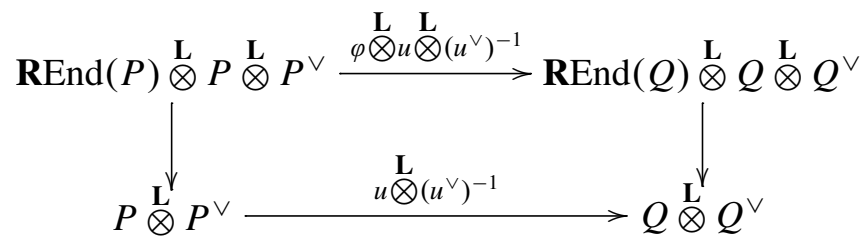

also commutes. Applying Lemma 5.1.2 and writing $B$ for $\operatorname{REnd}(Q)$, we find that the diagram

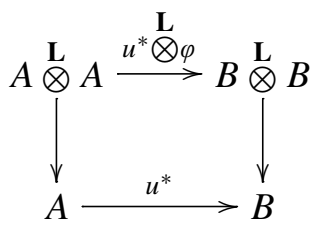

commutes, where the vertical arrows are the multiplication maps. Considering the units in the algebras, one readily concludes the proof of the claim. Note that to conclude that any such $u$ as above is an isomorphism, it suffices for its reduction 
to the residue field to be an isomorphism (for example, because the complexes are bounded above).

It is easy to see (using the realization in terms of diagrams of finite flat $\mathrm{O}$ modules) that $\operatorname{Hom}_{\mathbf{D}(\mathcal{O})}$ is compatible with flat base change and completion when restricted to the category of perfect complexes: given a flat ring extension $0 \rightarrow 0^{\prime}$, there is a natural isomorphism

$$
\operatorname{Hom}_{\mathbf{D}\left(\mathcal{O}^{\prime}\right)}\left(M \stackrel{\mathbf{L}}{\otimes} \mathcal{O}^{\prime}, N \stackrel{\mathbf{L}}{\otimes} \mathcal{O}^{\prime}\right) \cong \operatorname{Hom}_{\mathbf{D}(\mathcal{O})}(M, N) \otimes \mathcal{O}^{\prime}
$$

for all perfect $M$ and $N$ in $\mathbf{D}(0)$. Furthermore, given a perfect weak algebra $\Xi$, the realization of the module of $\Xi$-linear maps as a kernel of maps of Hom-modules shows that the same statement is true for Hom $\Xi$. Thus there is a commutative diagram

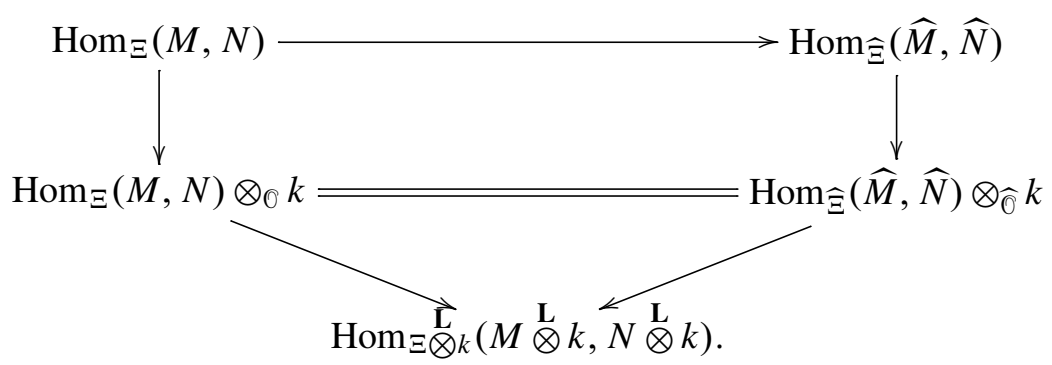

with surjective vertical arrows. This immediately applies to our situation to show that the map of Theorem 5.1.5 is surjective for $\widehat{O}$ if it is for $\widehat{0}$ (for a fixed $n$, which may be determined from the reduction to the residue field). Indeed, a $\widehat{\Xi}$-linear map $\widehat{M} \rightarrow \widehat{N}$ yields an element of $\operatorname{Hom}_{\widehat{\Xi}}(\widehat{M}, \widehat{N}) \otimes k$ whose image in the bottom module is an isomorphism. It follows from the diagram that there is a $\Xi$-linear map $u: M \rightarrow N$ whose (derived) reduction to $k$ is an isomorphism, whence $u$ is an isomorphism by Nakayama's lemma for perfect complexes (see, for example, [Lieblich 2006a, Lemma 2.1.3]).

Similarly, to verify that an isomorphism $\xi: P \stackrel{\sim}{\rightarrow} P$ in the kernel of the automorphism map is homotopic to a constant, it suffices to show that an element $\xi \in \operatorname{End}_{\mathbf{D}(\odot)}(P)$ is in $O_{P}$ if and only if this is true after completing. But the module of maps homotopic to a constant is also clearly compatible with flat base change and completion is moreover faithfully flat (all modules involved are finite over 0 because the complexes involved are perfect), so $\xi$ is in a submodule $Z$ of $\operatorname{End}(P)$ if and only if its image in $\operatorname{End}(P) \otimes \widehat{0}$ is contained in $Z \otimes \widehat{0}$.

From this point onward, we assume that $\mathbb{O}$ is a complete local Noetherian ring. Recall that a quotient of local rings $0 \rightarrow I \rightarrow 0 \rightarrow \overline{0} \rightarrow 0$ is small if $I$ is generated by an element $\varepsilon$ which is annihilated by the maximal ideal of 0 (so that, in particular, $\left.\varepsilon^{2}=0\right)$. We can choose a filtration $\mathcal{O} \supset \mathfrak{m}=I_{0} \supset I_{1} \supset I_{2} \supset \cdots$ which is separated 
(i.e., so that $\cap_{i} I_{i}=0$ ) and defines a topology equivalent to the $\mathfrak{m}$-adic topology such that for all $i \geq 0$, the quotient $0 \rightarrow I_{i} / I_{i+1} \rightarrow 0 / I_{i+1} \rightarrow 0 / I_{i} \rightarrow 0$ is a small extension, with $I_{i} / I_{i+1}$ generated by $\varepsilon_{i}$. We fix such a filtration for remainder of this section, and we denote $\mathbb{O} / I_{n}$ by $\mathcal{O}_{n}$.

Lemma 5.1.9. Let $0 \rightarrow I \rightarrow R \rightarrow \bar{R} \rightarrow 0$ be a surjection of rings. Let $A$ be a weak $R$-algebra and $P$ and $Q$ two left A-modules. Let $T$ denote the triangle in $\mathbf{D}(R)$ arising from the quotient map $R \rightarrow \bar{R}$.

(i) The maps in $P \stackrel{\mathbf{L}}{\otimes} T$ are A-linear (with the natural A-module structures).

(ii) Any A-linear map $\psi: P \rightarrow Q \stackrel{\mathbf{L}}{\otimes} \bar{R}$ factors through an A-linear map

$$
\bar{\psi}: P \stackrel{\mathbf{L}}{\otimes} \bar{R} \rightarrow Q \stackrel{\mathbf{L}}{\otimes} \bar{R}
$$

which is the derived restriction of scalars of an $A \stackrel{\mathbf{L}}{\otimes}$-linear map from $P \otimes \overrightarrow{\mathbf{L}} \bar{R}$ to $Q \stackrel{\mathbf{L}}{\otimes} \bar{R}$.

(iii) If $R \rightarrow \bar{R}$ is a small extension of local rings with residue field $k$, then the natural identification $P \stackrel{\mathrm{L}}{\otimes} \stackrel{\sim}{\rightarrow} P_{k}$ induced by a choice of basis for I over $k$ is A-linear.

Proof. Note that basic results about homotopy colimits allow us to replace any object of $\mathbf{D}(R)$ by a complex of projectives, so there are no boundedness conditions on any of the complexes involved. Part (i) follows immediately from the fact that $P \otimes(\cdot)$ is a functor from $\mathbf{D}(R)$ to $A$-modules. Part (ii) follows from writing $P$ and $A$ as complexes of projectives and representing the map $P \rightarrow Q \otimes \bar{R}$ as a map on complexes. (Note that this factorization need not be unique as a map in $\mathbf{D}(R)$, but it is unique as the derived restriction of scalars from a map in $\mathbf{D}(\bar{R})$.) Part (iii) follows similarly from looking at explicit representatives of $P$ and $A$.

Lemma 5.1.10. Suppose $f, g: P \rightarrow Q$ are two maps of perfect complexes in $K(\mathcal{O})$. Let $P_{n}=P \otimes \mathcal{O}_{n}, Q_{n}=Q \otimes \mathcal{O}_{n}, f_{n}=f \otimes \mathcal{O}_{n}, g_{n}=g \otimes \mathcal{O}_{n}$. Suppose there are homotopies

$$
h(n) \in \prod_{t} \operatorname{Hom}\left(P^{t}, Q^{t-1} \otimes I_{n}\right)
$$

such that for all $n$,

$$
f_{n}-g_{n}=d\left(\sum_{s<n} \bar{h}(s)\right)+\left(\sum_{s<n} \bar{h}(s)\right) d
$$

as maps of complexes, where $\bar{h}$ denotes the induced map. Then $f$ is homotopic to $g$.

Proof. The element $h=\sum_{s=0}^{\infty} h(s)$ converges and defines the homotopy. 
Lemma 5.1.11. Let $0 \rightarrow I \rightarrow R \rightarrow \bar{R} \rightarrow 0$ be a small extension of local rings with residue field $k$. Let $P$ and $Q$ be perfect complexes of $R$-modules (with chosen realizations) and $\varphi: \mathbf{R E n d}(P) \rightarrow \mathbf{R E n d}(Q)$ an isomorphism of the derived endomorphism algebras, written as a map in that direction on the underlying complexes. If there exists an isomorphism of the underlying complexes $\bar{u}: \bar{P} \stackrel{\sim}{\rightarrow}$ such that $\bar{\varphi}=\bar{u}^{*}$ as maps of complexes, then there is a lift $u$ of $\bar{u}$ and a homotopy $h$ between $\varphi$ and $u^{*}$ such that $h(\mathbf{R E n d}(P)) \subset \mathbf{R E n d}(Q) \otimes I$. In particular, $\varphi=u^{*}$ in $\mathbf{D}(R)$.

Proof. Let $A=\operatorname{REnd}(P)$ and let $A$ act on $Q$ via $\varphi$. The identification of $\bar{\varphi}$ with $\bar{u}^{*}$ provides an $\bar{A}$-linear isomorphism $\bar{\gamma}: \bar{P} \rightarrow \bar{Q}$, and we wish to lift this to an $A$-linear isomorphism $P \rightarrow Q$. Consider the composition $P \rightarrow \bar{Q} \rightarrow Q \otimes I(1) \cong Q_{k}(1)$ in the derived category. By Lemma 5.1.9, this map is $A$-linear and factors through an $A$-linear map $\alpha: P_{k} \rightarrow Q_{k}(1)$ which comes by derived restriction of scalars from an $A_{k}$-linear map in $\mathbf{D}(k)$. By Proposition 5.1.7 (and the method of its proof), we see that $\alpha$ is either zero or an isomorphism. But $P_{k} \cong Q_{k} ¥ 0$, which implies that $\alpha=0$. This means that there is an $R$-linear lift $\gamma$ of $\bar{\gamma}$. Now $\left(\gamma^{*}\right)^{-1} \circ \varphi-$ id is identified with a map $\operatorname{REnd}_{k}\left(P_{k}\right) \rightarrow \operatorname{REnd}_{k}\left(P_{k}\right)$ in $\mathbf{D}(k)$ which is a derivation of the algebra, hence is homotopic to the inner derivation induced by a map $\omega_{k}: P_{k} \rightarrow P_{k}$ in $\mathbf{D}(k)$. Writing $\omega$ for the composition

$$
P \longrightarrow P_{k} \stackrel{\omega_{k}}{\longrightarrow} P_{k} \stackrel{\cong}{\longrightarrow} P \stackrel{\mathbf{L}}{\otimes} I \longrightarrow P,
$$

we see that there exists a homotopy between $\varphi$ and $\gamma(1+\omega)^{*}$ having image in $\operatorname{REnd}(Q) \otimes I$, and that $\gamma(1+\omega)$ is a lift of $\bar{\gamma}$ as maps of complexes.

Lemma 5.1.12. Let $0 \rightarrow I \rightarrow R \rightarrow \bar{R} \rightarrow 0$ be a small extension of local rings with residue field $k$. Let $P$ be a perfect complex of $R$-modules (with a chosen realization) and $\psi: P \rightarrow P$ an automorphism of the underlying complex such that $\bar{\psi}=\bar{\alpha}$ for some $\bar{\alpha} \in \bar{R}_{\bar{P}}$ as maps of the complex $\bar{P}$ and such that $\psi^{*}$ is homotopic to the identity as a map of weak algebras. Then there is a unit $\alpha$ lifting $\bar{\alpha}$ and a homotopy $h$ between $\psi$ and $\alpha$ such that $h(\mathbf{R E n d}(P)) \subset \mathbf{R E n d}(P) \otimes I$.

Proof. The proof is quite similar to the proof of Lemma 5.1.11, using the left half of the exact sequence of Corollary 5.1.6 rather than the right half.

Proposition 5.1.13. Theorem 5.1.5 holds for $O$ (now assumed complete).

Proof. Given an isomorphism $\varphi: \operatorname{REnd}(P) \stackrel{\sim}{\rightarrow} \operatorname{REnd}(Q)$, we may assume after adding zero complexes to $P$ and $Q$, shifting $Q$, and applying a homotopy to $\varphi$, that there is an isomorphism $\psi_{0}: P_{0} \rightarrow Q_{0}$ such that $\varphi_{0}=\psi_{0}^{*}$ as maps of complexes. We can now apply Lemma 5.1.11 to arrive at an isomorphism $\psi_{1}$ lifting $\psi_{0}$ and a homotopy $\bar{h}(0)$ with image in $\operatorname{REnd}_{\mathscr{O}_{1}}\left(Q_{1}\right) \otimes_{\mathbb{O}_{1}} I_{0} / I_{1}$ between $\varphi_{1}$ and $\psi_{1}^{*}$. Lift $\bar{h}(0)$ to a homotopy $h(0)$ with image in $\operatorname{REnd}(Q) \otimes I_{0}$. Then

$$
(\varphi-(d h(0)+h(0) d))_{1}=\psi_{1}^{*}
$$


as maps of complexes, and we may find a homotopy $h(1)$, etc. By Lemma 5.1.10, we see that there is an isomorphism $\psi: P \rightarrow Q$ such that $\varphi=\psi^{*}$ in $\mathbf{D}(0)$. A similar argument shows that the kernel is $\mathrm{O}_{P}^{*}$.

5.2. The construction of GAz. In this section, we define a stack which we will use to compactify the stack of Azumaya algebras. While the definition is rather technical in general, in the case of a relative surface it assumes a simpler and more intuitive form.

Let $(X, O)$ be a ringed topos.

Definition 5.2.1. A pregeneralized Azumaya algebra on $X$ is a perfect algebra object $\mathscr{A}$ of the derived category $\mathbf{D}(X)$ of $\mathrm{O}_{X}$-modules such that there exists an object $U \in X$ covering the final object and a totally supported perfect sheaf $\mathscr{F}$ on $U$ with $\left.\mathscr{A}\right|_{U} \cong \mathbf{R} \mathscr{E} d_{U}(\mathscr{F})$ as weak algebras. An isomorphism of pregeneralized Azumaya algebras is an isomorphism in the category of weak algebras.

5.2.1. Stackification. Consider the fibered category $\mathscr{P} \mathscr{R} \rightarrow S c h$ ét of pregeneralized Azumaya algebras on the large étale topos over $\operatorname{Spec} \mathbb{Z}$. We will stackify this to yield the stack of generalized Azumaya algebras. This is slightly different from the construction given in [Laumon and Moret-Bailly 2000, 3.2], as we do not assume that the fibered category is a prestack.

Lemma 5.2.1.1. Suppose $T$ is a topos and $\mathscr{C} \rightarrow T$ is a category fibered in groupoids. There exists a stack $\mathfrak{C}^{s}$, unique up to 1-isomorphism, and a 1-morphism $\mathfrak{b}_{\rightarrow} \mathscr{C}^{s}$ which is universal among 1-morphisms to stacks (up to 2-isomorphism).

Proof. The proof is the usual type of argument. A reader interested in seeing a generalization to stacks in categories larger than groupoids should consult [Giraud 1971]. First, we may assume that in fact $\mathscr{C} \rightarrow T$ admits a splitting (after replacing $\mathscr{C}$ by a 1 -isomorphic fibered category). Define a new fibered category $\mathscr{C}^{p}$ as follows: the objects will be the same, but the morphisms between two objects $a$ and $b$ over $t \in T$ will be the global sections of the sheafification of the presheaf $\operatorname{Hom}_{t}(a, b)$ : $(s \stackrel{\varphi}{\rightarrow} t) \mapsto \operatorname{Hom}_{s}\left(\varphi^{*} a, \varphi^{*} b\right)$ on $t$. Clearly $\mathscr{C}^{p}$ is a prestack (that is, given any two sections $a$ and $b$ over $t$, the hom-presheaf just described is a sheaf) and the natural map $\mathscr{C} \rightarrow \mathscr{C}^{p}$ of fibered categories over $T$ is universal up to 1 -isomorphism for 1 -morphisms of $\mathscr{C}$ into prestacks. Now we apply [Laumon and Moret-Bailly 2000, 3.2] to construct $\mathscr{C}^{s}$ as the stackification of $\mathscr{C}^{p}$.

Definition 5.2.1.2. The stack of generalized Azumaya algebras on schemes is defined to be the stack in groupoids $\mathscr{P} \mathscr{R}^{s} \rightarrow S c h_{\text {ét }}$ associated to the fibered category of pregeneralized Azumaya algebras.

Remark 5.2.1.3. Explicitly, given a scheme $X$, to give a generalized Azumaya algebra on $X$ is to give an étale 3-hypercovering $Y^{\prime \prime} \Longrightarrow Y^{\prime} \Longrightarrow Y \longrightarrow X$, 
a totally supported sheaf $\mathscr{F}$ on $Y$, and a gluing datum for $\mathbf{R} \mathscr{E} n d_{Y}(\mathscr{F})$ in the derived category $\mathbf{D}\left(Y^{\prime}\right)$ whose coboundary in $\mathbf{D}\left(Y^{\prime \prime}\right)$ is trivial. Two such objects $\left(Y_{1}, \mathscr{F}_{1}, \delta_{1}\right)$ and $\left(Y_{2}, \mathscr{F}_{2}, \delta_{2}\right)$ are isomorphic if and only if there is a common refinement $Y_{3}$ of the 3-hypercovers $Y_{1}$ and $Y_{2}$ and an isomorphism $\varphi:\left.\left.\mathscr{F}_{1}\right|_{Y_{3}} \stackrel{\sim}{\rightarrow} \mathscr{F}_{2}\right|_{Y_{3}}$ commuting with the restrictions of $\delta_{1}$ and $\delta_{2}$. Thus, a generalized Azumaya algebra is gotten by gluing "derived endomorphism algebras" together in the étale topology. When $X$ is a quasiprojective smooth surface, or, more generally, a quasiprojective scheme smooth over an affine with fibers of dimension at most 2 , then the sections of $\mathscr{P} \mathscr{R}$ over $X$ are the same as the sections of $\mathscr{P}_{\mathscr{R}^{s}}$ over $X$; see Section 6.4.

Example 5.2.1.4. Let $\pi: \mathscr{X} \rightarrow X$ be a $\boldsymbol{\mu}_{n}$-gerbe and $\mathscr{F}$ a totally supported perfect $\mathscr{L}$-twisted sheaf. The complex $\mathbf{R} \pi_{*} \mathbf{R}_{\mathscr{E}}^{\mathscr{E}} d_{\mathscr{L}}(\mathscr{F}) \in \mathbf{D}(X)$ is a pregeneralized Azumaya algebra, hence has a naturally associated generalized Azumaya algebra. We will see below that the global sections of the stack $\mathscr{P} \mathscr{R}^{s}$ are precisely the weak algebras of this form.

Lemma 5.2.1.5. Let $\mathscr{F}$ and $G$ be totally supported perfect sheaves on a $\mathbf{G}_{m}$-gerbe $\mathscr{X} \rightarrow X$.

(1) The sheaf of isomorphisms between the generalized Azumaya algebras as-

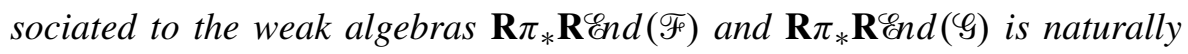
isomorphic to $\operatorname{Isom}(\mathscr{F}, \mathscr{G}) / \mathbf{G}_{m}$, with $\mathbf{G}_{m}$ acting by scalar multiplication on $\mathscr{G}$.

(2) Any isomorphism of generalized Azumaya algebras

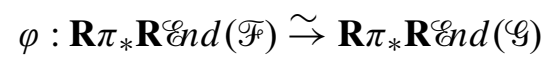

is the isomorphism associated to an isomorphism $\mathscr{F} \stackrel{\sim}{\rightarrow} \mathscr{L} \otimes \mathscr{G}$ for some invertible sheaf $\mathscr{L}$ on $X$.

Proof. Temporarily write $\mathscr{I}$ for the sheaf of isomorphisms of generalized Azumaya

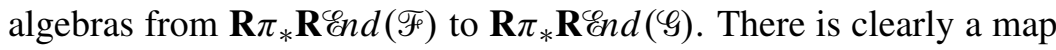

$$
\chi: \operatorname{Isom}(\mathscr{F}, \mathscr{G}) / \mathbf{G}_{m} \rightarrow \mathscr{I} .
$$

To verify that it is an isomorphism, it suffices to verify it étale-locally on $X$, whence we may assume that $X$ is strictly local. Choosing an invertible $\mathscr{L}$-twisted sheaf and twisting down $\mathscr{F}$ and $\mathscr{G}$, we are reduced to showing the analogous statement for totally supported sheaves on $X$ itself. Any local section of $\mathscr{I}$ comes from an isomorphism of weak algebras $\mathbf{R}_{\mathscr{E}}^{\mathscr{E}} \mathrm{n}(\mathscr{F}) \stackrel{\sim}{\rightarrow} \mathbf{R}_{\mathscr{E}}^{\mathscr{E}} \mathrm{d}(\mathscr{G})$, so Theorem 5.1.5 shows that $\chi$ is surjective. Similarly, any section of the kernel of $\chi$ must locally be trivial, whence $\chi$ is an isomorphism.

The second part is a formal consequence of the first: there is an étale covering $U \rightarrow X$ such that $\left.\varphi\right|_{U}$ is associated to an isomorphism $\psi:\left.\left.\mathscr{F}\right|_{U} \stackrel{\sim}{\rightarrow} \mathscr{G}\right|_{U}$. The coboundary of $\psi$ on the product $U \times{ }_{X} U$ is multiplication by some scalar, which 
is a cocycle by a formal calculation. This gives rise to the invertible sheaf $\mathscr{L}$; tensoring with $\mathscr{L}$ makes the coboundary of $\psi$ trivial, whence it descends to an isomorphism $\mathscr{F} \rightarrow \mathscr{L} \otimes \mathscr{G}$ inducing $\varphi$, as desired.

Definition 5.2.1.6. Let $\mathscr{A}$ be a generalized Azumaya algebra on $X$. The gerbe of trivializations of $\mathscr{A}$, denoted $\mathscr{X}(\mathscr{A})$, is the stack on the small étale site $X_{\text {ét }}$ whose sections over $V \rightarrow X$ given by pairs $(\mathscr{F}, \varphi)$, where $\mathscr{F}$ is a totally supported sheaf on $V$ and $\varphi:\left.\mathbf{R}_{\mathscr{E}}^{\mathscr{E}} d_{V}(\mathscr{F}) \stackrel{\sim}{\rightarrow} \mathscr{A}\right|_{V}$ is an isomorphism of generalized Azumaya algebras. The isomorphisms in the fiber categories are isomorphisms of the sheaves which respect the identifications with $\mathscr{A}$, as usual.

This is entirely analogous to the gerbe produced in [Giraud 1971, section V.4.2]. There is also an analogue of the $\boldsymbol{\mu}_{n}$-gerbe associated to an Azumaya algebra of degree $n$.

Definition 5.2.1.7. Given a generalized Azumaya algebra $\mathscr{A}$ of degree $n$ on $\mathscr{X}$, the gerbe of trivialized trivializations of $\mathscr{A}$, denoted $\mathscr{X}_{\text {triv }}(\mathscr{A})$, is the stack on the small étale site of $X$ whose sections over $U \rightarrow X$ consist of triples $(\mathscr{F}, \varphi, \delta)$ with $\varphi: \mathbf{R}_{\mathscr{C}}^{\mathscr{n}} n d_{U}(\mathscr{F}) \stackrel{\sim}{\rightarrow} \mathscr{A}_{U}$ an isomorphism of generalized Azumaya algebras and $\mathrm{O}_{U} \stackrel{\sim}{\rightarrow} \operatorname{det}(\mathscr{F})$ an isomorphism of invertible sheaves on $U$. The isomorphisms in the fiber categories are isomorphisms of the sheaves which preserve the identifications with $\mathscr{A}$ and the trivializations of the determinants.

Lemma 5.2.1.8. The stack $\mathscr{X}(\mathscr{A})$ is a $\mathbf{G}_{m}$-gerbe. If $\mathscr{A}$ has degree $n$, then $\mathscr{X}_{\text {triv }}(\mathscr{A})$ is a $\boldsymbol{\mu}_{n}$-gerbe whose associated cohomology class maps to $[\mathscr{Q}(\mathscr{A})]$ in $\mathrm{H}^{2}\left(X, \mathbf{G}_{m}\right)$.

Proof. This follows immediately from the derived Skolem-Noether Theorem 5.1.5 and the fact that all of the sheaves $\mathscr{F}$ are totally supported.

Corollary 5.2.1.9. A generalized Azumaya algebra $A$ has a class in $\mathrm{H}^{2}\left(X, \mathbf{G}_{m}\right)$. When the rank of $\mathscr{A}$ is $n^{2}$, A has a class in $\mathrm{H}^{2}\left(X, \boldsymbol{\mu}_{n}\right)$ (in the fppf topology).

Definition 5.2.1.10. When rk $\mathscr{A}=n^{2}$, we call the cohomology class in $\mathrm{H}^{2}\left(X, \boldsymbol{\mu}_{n}\right)$ the class of $\mathscr{A}$, and write $\operatorname{cl}(\mathscr{A})$.

Let $\pi: \mathscr{L}(\mathscr{A}) \rightarrow X$ denote the natural projection.

Lemma 5.2.1.11. There is an $\mathscr{X}(\mathscr{A})$-twisted sheaf $\mathscr{F}$ and an isomorphism of generalized Azumaya algebras $\varphi: \mathbf{R} \pi_{*} \mathbf{R}_{\mathscr{C}}^{\mathscr{n}} d_{\mathscr{L}(\mathscr{A})}(\mathscr{F}) \stackrel{\sim}{\rightarrow} \mathscr{A}$. The datum $(\mathscr{X}(\mathscr{A}), \mathscr{F}, \varphi)$ is functorial in A.

Proof. As usual, the construction of $\mathscr{L}(\mathscr{A})$ yields by first projection a twisted sheaf $\mathscr{F}$. Whenever $\mathscr{L}(\mathscr{A})$ has a section $f$ over $V$, there is by construction an isomorphism $\left.\mathbf{R}^{\mathscr{E}} n d_{V}\left(f^{*} \mathscr{F}\right) \rightarrow \mathscr{A}\right|_{V}$, and this is natural in $V$ and $f$. This is easily seen to imply the remaining statements of the lemma.

Let $\mathscr{D} \rightarrow S c h_{\text {ét }}$ denote the fibered category of derived categories which to any scheme $X$ associates the derived category $\mathbf{D}(X)$ of étale $\mathbb{O}_{X}$-modules. 
Proposition 5.2.1.12. There is a faithful morphism of fibered categories $\mathscr{P} \mathscr{R}^{s} \rightarrow \mathscr{D}$ which identifies $\mathscr{P} \mathscr{R}^{s}$ with the subcategory of $\mathscr{D}$ whose sections over $X$ are weak algebras of the form $\mathbf{R} \pi_{*} \mathbf{R}_{\mathscr{E}} d_{\mathscr{X}}(\mathscr{F})$, where $\pi: \mathscr{X} \rightarrow X$ is a $\mathbf{G}_{m}$-gerbe, and whose isomorphisms $\mathbf{R} \pi_{*} \mathbf{R}_{\mathscr{E}}^{\bullet} d_{\mathscr{X}}(\mathscr{F}) \stackrel{\sim}{\rightarrow} \mathbf{R} \pi_{*}^{\prime} \mathbf{R}^{\complement} \mathrm{Ed}_{\mathscr{X}^{\prime}}\left(\mathscr{F}^{\prime}\right)$ are naturally a pseudotorsor un$\operatorname{der} \operatorname{Aut}(\mathscr{F}) / \mathbf{G}_{m}$.

Proof. The morphism $\mathscr{P} \mathscr{R}^{s} \rightarrow \mathscr{D}$ comes from Lemma 5.2.1.11. Given $\mathscr{A}$ and $\mathscr{B}$, an isomorphism $\varphi: \mathscr{A} \rightarrow \mathscr{B}$ induces an isomorphism $\mathscr{X}(\mathscr{A}) \stackrel{\sim}{\rightarrow} \mathscr{X}(\mathscr{B})$. Thus,

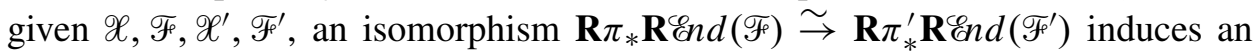
isomorphism $\varepsilon: \mathscr{X} \rightarrow \mathscr{X}^{\prime}$ and an isomorphism of generalized Azumaya algebras

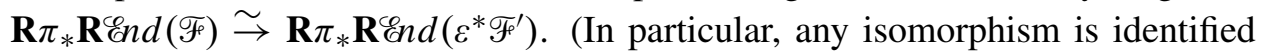
with an isomorphism of the underlying weak algebras.) By Lemma 5.2.1.5, once there is an isomorphism the set of isomorphisms is a torsor under $\mathscr{A} u t(\mathscr{F}) / \mathbf{G}_{m}$, as claimed. The faithfulness also results from Lemma 5.2.1.5.

Remark 5.2.1.13. When $\mathscr{X}=\mathscr{X}^{\prime}$ in Proposition 5.2.1.12, the sheaf of isomorphisms is simply identified with the quotient sheaf $\operatorname{Isom}\left(\mathscr{F}_{\mathscr{F}}, \mathscr{F}^{\prime}\right) / \mathbf{G}_{m}$. This will be the case when we study the moduli of generalized Azumaya algebras on a surface, as the (geometric) Brauer class will be constant in families.

Thus, at the end of the complex process of stackification, one is left simply with the derived endomorphism algebras of twisted sheaves, with a subset of the quasiisomorphisms giving the isomorphisms.

5.2.2. Identification with rigidifications. Let $\mathscr{G}_{X}(n)$ be the stack of generalized Azumaya algebras on $X$ of degree $n$.

Proposition 5.2.2.1. The morphism $\rho: \mathscr{T}_{X}^{\text {parf }} \rightarrow \mathscr{G}_{X}$ sending $\mathscr{F}$ to $\mathbf{R}^{\mathscr{E}}$ nd $(\mathscr{F})$ yields an isomorphism $\mathscr{T}_{X} / \mathbf{G}_{m} \stackrel{\sim}{\rightarrow} \mathscr{G}_{X}$.

Proof. It follows from Theorem 5.1.5 that $\mathscr{G}_{X}$ is the stackification of the prestack given by taking totally supported sheaves and replacing $\operatorname{Isom}(\mathscr{F}, \mathscr{G})$ with $\operatorname{Isom}\left(\mathscr{F}_{,}, \mathscr{G}\right) / \mathbf{G}_{m}$. But this is precisely how $\mathscr{T}_{X}^{\text {parf }} / \mathbf{G}_{m}$ is constructed!

Lemma 5.2.2.2. If $2 \rightarrow 2^{\prime}$ is a morphism of prestacks on a site which is fully faithful on fiber categories and an epimorphism (that is, any object of $2^{\prime}$ is locally in the image of 2) then the induced map of stackifications is an isomorphism.

Proof. An object of the stackification is just an object of the prestack with a descent datum. Moreover, refining the descent datum yields a naturally isomorphic object of the stackification. Thus, we see that the map on stackifications $\widetilde{\mathscr{2}} \rightarrow \widetilde{\mathscr{2}^{\prime}}$ is fully faithful and an epimorphism. (Indeed, after refining the descent datum on an object of $2^{\prime}$, we can assume the object and descent datum come from 2.) It follows that it must be an isomorphism. 
Proposition 5.2.2.3. Assume $n$ is invertible on $X$. The morphism

$$
\rho: \mathscr{T}_{X}^{\mathbb{O}}(n) \rightarrow \mathscr{G}_{X}(n)
$$

sending $\mathscr{F}$ to $\mathbf{R}^{\mathscr{E}}$ nd $(\mathscr{F})$ yields an isomorphism $\mathscr{T}_{X}^{0} \| \boldsymbol{\mu}_{n} \stackrel{\sim}{\rightarrow} \mathscr{G}_{X}$.

Proof. In light of Proposition 5.2.2.1, it is enough to prove that the natural map $\varphi: \mathscr{T}_{X}^{\mathbb{O}}(n) \rightarrow \mathscr{T}_{X}(n)$ yields an isomorphism of the appropriate rigidifications. But $\varphi$ is clearly an epimorphism. Further, for any $\mathscr{F}$ and $\mathscr{G}$ with trivialized determinants, $\varphi$ induces an isomorphism of sheaves $f: \operatorname{Isom}_{\operatorname{det}}(\mathscr{F}, \mathscr{G}) / \boldsymbol{\mu}_{n} \stackrel{\sim}{\rightarrow} \operatorname{Isom}(\mathscr{F}, \mathscr{G}) / \mathbf{G}_{m}$. To check this, it is enough to suppose $X=\operatorname{Spec} A$ is strictly Henselian. Since $n$ is invertible on $X$, any unit of $A$ has an $n$-th root, from which it follows that $f$ is surjective. If $\gamma: \mathscr{F} \rightarrow \mathscr{G}$ and $\eta: \mathscr{F} \rightarrow \mathscr{G}$ are isomorphisms which preserve determinants and differ by multiplication by a scalar $\theta$ on $\varphi$ then $\theta$ must be an $n$-th root of unity, which shows that $f$ is injective.

Forming prestacks by dividing out by the appropriate scalars, we thus find a morphism of prestacks $2 \rightarrow 2^{\prime}$ which is fully faithful on fiber categories and is an étale epimorphism. Applying Lemma 5.2.2.2 completes the proof.

5.2.3. The relative case. Now we push everything forward (with one important Warning 5.2.3.2 below) to define relative stacks of generalized Azumaya algebras.

Definition 5.2.3.1. Let $f: X \rightarrow S$ be a morphism. A relative generalized Azumaya algebra on $X / S$ is a generalized Azumaya algebra on $X$ whose local sheaves are $S$-flat and totally pure in each geometric fiber. This is equivalent to writing $\mathscr{A} \cong$ $\mathbf{R} \pi_{*} \mathbf{R} \mathscr{E} d_{\mathscr{X}}(\mathscr{F})$ with $\mathscr{X} \rightarrow X$ a $\mathbf{G}_{m}$-gerbe and $\mathscr{F}$ an $S$-flat $\mathscr{L}$-twisted sheaf which is totally pure in every geometric fiber.

Warning 5.2.3.2. Even though the absolute theory of generalized Azumaya algebras used totally supported sheaves, in the relative theory we will use totally pure sheaves. While this is not necessary for the abstract results to be true, it gives a better moduli theory when $X / S$ is sufficiently nice (for example, a smooth projective surface).

As in Definition 5.2.1.6, one may define the class of such a generalized Azumaya algebra. Let $\mathscr{X} \rightarrow X$ be a fixed $\mu_{n}$-gerbe, with $n \in \mathcal{O}_{S}(S)^{\times}$.

Notation 5.2.3.3. Let $\mathbf{G A z _ { \mathscr { X } } / S}(n)$ denote the stack of generalized Azumaya algebras on $X / S$ of rank $n^{2}$ in every geometric fiber whose class agrees with [ étale locally around every point on the base. When we do not wish to specify the cohomology class, we will write $\mathbf{G A z}_{X / S}(n)$ for the stack of generalized Azumaya algebras of rank $n^{2}$ on each fiber.

When $X \rightarrow S$ is proper and $n$ is invertible on $S$, the condition that the cohomology class agree with $[\mathscr{X}$ ] étale-locally on $S$ is equivalent to the condition that it agree with $[\mathscr{X}]$ in every geometric fiber. 
5.2.4. Identification of $\mathbf{G A z} \mathbf{z}_{\mathscr{X} / S}(n)$ with $\mathcal{M}_{n}^{\mathscr{X}}$. Let $\mathscr{G}_{X / S}(n)$ be the stack on $X_{\text {rét }}$ parametrizing generalized Azumaya algebras that are locally isomorphic to $\mathbf{R}_{\mathscr{E}}^{\mathscr{E}} \mathrm{n} d(\mathscr{F})$ with $\mathscr{F}$ an object of $\mathscr{P}_{X / S}^{\mathrm{parf}}(n)$. An argument identical to Proposition 5.2.2.1 shows that the natural map $\mathscr{P}_{X / S}^{\mathbb{O}}(n) \rightarrow \mathscr{G}_{X / S}(n)$ yields an isomorphism

$$
\mathscr{P}_{X / S}^{O}(n) \| \boldsymbol{\mu}_{n} \stackrel{\sim}{\rightarrow} \varphi_{X / S}(n) .
$$

On the other hand, it is easy to see that $\mathbf{G A z}_{X / S}(n)=f_{*}\left(\mathscr{G}_{X / S}(n)\right)$ and that $\mathbf{G A z}_{\mathscr{L} / S}(n)=f_{*}^{\mathscr{X}}\left(\varphi_{X / S}(n)\right)$. We conclude that $\mathbf{G A z} \mathscr{\mathscr { L }} / S(n) \cong \mathcal{M}_{n}^{\mathscr{X}}$, thus showing that generalized Azumaya algebras give a coherent model for $\mathcal{M}_{n}^{\mathscr{X}}$. Moreover, it is easy to see that $M_{n}^{\mathscr{L}}(c)$ is identified with the stack of generalized Azumaya algebras of the form $\mathbf{R} \mathscr{H} \operatorname{lom}(\mathscr{F})$ with $\mathscr{F}$ an $\mathscr{X}$-twisted sheaf with trivialized determinant and $\operatorname{deg} c_{2}(\mathscr{F})=c / 2 n$. This condition is equivalent to the condition that $\operatorname{deg} c_{2}\left(\mathbf{R}^{\mathscr{E}} n d(\mathscr{F})\right)=c$, giving a coherent interpretation of $\mathcal{M}_{n}^{\mathscr{X}}(c)$.

For the reader uncomfortable with the stackification procedure (in spite of its concrete outcome), we will show in Section 6.4 that when $X$ is a surface stackification is in fact unnecessary.

\section{Moduli of stable $\mathrm{PGL}_{n}$-torsors on surfaces}

For the rest of this paper, we assume that $S=\operatorname{Spec} k$ with $k$ a separably closed field and $X / S$ a smooth projective surface with a fixed ample divisor $H$.

6.1. Stability of torsors. We first recall a basic definition.

Definition 6.1.1. Given a torsion-free sheaf $\mathscr{F}$, the slope of $\mathscr{F}$ is $\operatorname{deg} \mathscr{F} / \mathrm{rk} \mathscr{F}$.

To define stability for $\mathrm{PGL}_{n}$-torsors, we use the adjoint sheaf. As described in Section 3.3, this adjoint sheaf naturally comes with an algebra structure, which we will use in our definition.

Definition 6.1.2. An Azumaya algebra $\mathscr{A}$ on $X$ is stable if for all nonzero right ideals $\mathscr{I} \subset \mathscr{A}$ of rank strictly smaller than rk $\mathscr{A}$ we have $\mu(\mathscr{T})<0$.

Remark 6.1.3. It is equivalent to quantify over left ideals. Thus, one could state the definition by omitting the word "right" and quantifying over arbitrary ideals, understood as right or left ideals. It is of course not sufficient to quantify over two-sided ideals.

Remark 6.1.4. This definition is meant to apply only to the classical notion of slope-stability, and not to the more refined notion due to Gieseker. While such notions of stability using normalized Hilbert polynomials are essential for the development of moduli theory using geometric invariant theory (GIT), they are somewhat artificial in the sense that they no longer correspond to the existence of a Hermite-Einstein connection. (However, recent work of Wang [2002; 2005] 
has clarified the analytic meaning of Gieseker-stability in terms of the existence of certain weak Hermite-Einstein connections.)

One way to understand the compactifications of the stack of slope-stable bundles — using slope-semistability or Gieseker-semistability, GIT-bound or purely stacky, etc. - is that each really only serves to impose the kind of inductive strucure on the moduli problem necessary to prove theorems about the actual part of interest: the open sublocus of slope-stable bundles. Working in a GIT-free manner (which is necessary in the context of twisted sheaves) frees us to ignore the subtleties (both algebraic and analytic) of Gieseker-stability. This is pursued in [Lieblich 2008a], where the asymptotic properties of moduli are proved entirely without GIT.

6.1.5. To relate Definition 6.1.2 to the cover of $f_{*}\left(\mathrm{BPGL}_{n}\right)$ by the stack of twisted sheaves, we recall some rudiments from the theory of Chern classes for twisted sheaves. A different development of the theory of Chern classes for twisted sheaves and the relationship to the theory described here is given in [Heinloth 2005].

Given a coherent $\mathscr{X}$-twisted sheaf $\mathscr{F}$, we can use the rational Chow theory of $\mathscr{X}$ to define Chern classes $c_{i}(\mathscr{F}), i=1,2$. (The first Chern class $c_{1}(\mathscr{F})$ is just the class in Chow theory associated to the invertible sheaf det $\mathscr{F}$.) There is also a degree map from $d: A_{0}(\mathscr{L}) \rightarrow \mathbb{Q}$; this has the property that the 0 -cycle supported over a closed point of $X$ has degree $1 / n$. We define a normalized degree function by deg $=n d$. Using this degree, we have the following definition.

Definition 6.1.6. A torsion-free $\mathscr{X}$ twisted sheaf $\mathscr{V}$ is stable if for every subsheaf $\mathscr{W} \subset \mathcal{V}$ we have

$$
\mu(\mathscr{W})<\mu(\mathscr{V}) .
$$

As a special case of [Lieblich 2007, 2.2.7.22], it follows that if $\mathscr{F}$ is a flat family of coherent $\mathscr{L}$-twisted sheaves parametrized by $T$ with trivialized determinant, then the function $t \mapsto \operatorname{deg} c_{2}\left(\mathscr{F}_{t}\right)$ is locally constant on $T$. Moreover, by [Lieblich 2008a, Proposition 4.3.1.2], we have that stability is an open condition in a flat family of torsionfree $\mathscr{X}$-twisted sheaves.

Notation 6.1.7. Let $\mathbf{T w}_{\mathscr{L} / k}(n, \mathcal{O}, c) \subset \mathbf{T w}_{\mathscr{X} / k}(n, \mathcal{O})$ denote the open and closed substack parametrizing families such that $\operatorname{deg} c_{2}\left(\mathscr{F}_{t}\right)=c$ in each geometric fiber. Let $\mathbf{T w}_{\mathscr{Q} / k}^{s}(n, \mathbb{O}, c) \subset \mathbf{T} \mathbf{w}_{\mathscr{L} / k}(n, \mathbb{O}, c)$ denote the open substack whose objects over $T$ are families $\mathscr{F}_{F}$ such that the fiber $\mathscr{F}_{t}$ is stable for each geometric point $t \rightarrow T$.

Lemma 6.1.8. A locally free $\mathscr{L}$-twisted sheaf $\mathscr{V}$ is stable if and only if the Azumaya algebra $\pi_{*} \stackrel{E}{\mathrm{E}} \mathrm{d}(\mathfrak{V})$ is stable.

Proof. Given a subsheaf $\mathscr{W} \subset \mathcal{V}$, a straightforward computation shows that

$$
\mu(\mathscr{H o m}(\mathscr{V}, \mathscr{W}))=\mu(\mathscr{W})-\mu(\mathscr{V}) .
$$


On the other hand, any right ideal of $\mathscr{A}$ has the form $\mathscr{H o m}(\mathscr{V}, \mathscr{W})$ for a subsheaf $\mathscr{W} \subset \mathcal{V}$. The result follows.

6.1.9. Suppose $B \rightarrow S$ is a $k$-scheme and $T \rightarrow X_{B}$ is a $\mathrm{PGL}_{n}$-torsor. Let $\mathscr{A}$ be the locally free sheaf (of rank $n^{2}$ ) associated to the adjoint torsor (which is a GL $n_{n^{2}}$ torsor). Using the Riemann-Roch theorem, the invariance of Euler characteristic in a flat family, and the fact that $\operatorname{det} \mathscr{A} \cong \mathbb{O}$, we see that the function $b \mapsto \operatorname{deg} c_{2}\left(\mathscr{A}_{b}\right)$ is locally constant on $B$. This provides a numerical invariant of a $\mathrm{PGL}_{n}$-torsor which is constant in a family. Given a $\boldsymbol{\mu}_{n}$-gerbe $\mathscr{X} \rightarrow X$ and an integer $c$, let $\left(f_{*}^{\mathscr{X}} \mathrm{BPGL}_{n}\right)(c)$ be the substack of $f_{*}^{\mathscr{X}} \mathrm{BPGL}_{n}$ parametrizing families where the locally free sheaf associated to the adjoint bundle has $\operatorname{deg} c_{2}=c$ in every fiber.

Thus, there is a decomposition

$$
f_{*} \mathrm{BPGL}_{n}=\sqcup_{\mathscr{L}} \sqcup_{c}\left(f_{*}^{\mathscr{X}} \mathrm{BPGL}_{n}\right)(c),
$$

where the first disjoint union is taken over a set of $\boldsymbol{\mu}_{n}$-gerbe representatives for $\mathrm{H}^{2}\left(X, \boldsymbol{\mu}_{n}\right)$ and the second is taken over $\mathbb{Z}$. Similarly, there is a decomposition

$$
f_{*}\left(\mathrm{BPGL}_{n}\right)^{s}=\sqcup_{\mathscr{X}} \sqcup_{c}\left(f_{*}^{\mathscr{X}}\left(\mathrm{BPGL}_{n}\right)^{s}\right.
$$

of stable loci.

Lemma 6.1.10. Given an integer $c$, the closed and open substack $\mathbf{T w}_{\mathscr{L} / S}(n, \mathcal{O}, c / 2 n)$ is equal to the preimage of its image in $\mathcal{M}_{n}^{\mathscr{Q}}$. Similarly, $\mathbf{T w}_{\mathscr{L} / k}^{S}(n, \mathcal{O}, c / 2 n)$ is equal to the preimage of its image in $M_{n}^{\mathscr{Q}}$.

Proof. Given a point $p$ of $\mathcal{M}_{n}^{\mathscr{R}}$ which lifts into [F्F] $\in \mathbf{T w}_{\mathscr{L} / S}(n, \mathcal{O}, c / 2 n)$, it is easy to see that the full preimage of $p$ in $\mathbf{T w}_{\mathscr{L} / S}(n, \mathcal{O})$ is given by the twists $\mathscr{F} \otimes \mathscr{L}$ with $\mathscr{L} \in \mathscr{P} i c_{X / S}[n]$. But these have the same (rational) Chern classes as $\mathscr{F}$, as $\mathscr{L}$ is trivial, so they also lie in $\mathbf{T w}_{\mathscr{X} / S}(n, \mathbb{O}, c / 2 n)$. The second statement follows from the fact that $\mathscr{F}$ is stable if and only if $\mathscr{F} \otimes \mathscr{L}$ is stable for an invertible sheaf $\mathscr{L}$.

Corollary 6.1.11. There is an open substack $f_{*}\left(\mathrm{BPGL}_{n}\right)^{s} \subset f_{*}\left(\mathrm{BPGL}_{n}\right)$ parametrizing families $P \rightarrow X_{T}$ of $\mathrm{PGL}_{n}$-torsors such that for all geometric points $t \rightarrow T$ the fiber $P_{t} \rightarrow X_{t}$ is a stable $\mathrm{PGL}_{n}$-torsor.

Proof. This follows from Lemma 6.1.10 together with the fact that $\mathbf{T w}_{\mathscr{L} / k}(n, \mathcal{O})^{\mathrm{lf}} \rightarrow$ $f_{*}\left(\mathrm{BPGL}_{n}\right)$ is universally submersive.

Hence, since $\mathbf{T w}_{\mathscr{X} / S}(n, \mathcal{O}, c / 2 n)$ is open and closed in $\mathbf{T w}_{\mathscr{L} / S}(n, \mathcal{O})$, there is a welldefined open and closed substack $M_{n}^{\mathscr{L}}(c)$ whose preimage is $\mathbf{T} \mathbf{w}_{\mathscr{L} / S}(n, \mathbb{O}, c / 2 n)$. There is an open substack $\mathcal{M}_{n}^{\mathscr{X}}(c)^{s}$ whose preimage is $\mathbf{T w}_{\mathscr{Q} / k}^{s}(n, \mathbb{O}, c / 2 n)$. Each $\mathcal{M}_{n}^{\mathscr{L}}(c)$ is quasiproper and there is an open immersion $f_{*}^{\mathscr{X}} \mathrm{BPGL}_{n}(c) \hookrightarrow \mathcal{M}_{n}^{\mathscr{Q}}(c)$ and an open immersion $f_{*}^{\mathscr{X}} \mathrm{BPGL}_{n}(c)^{s} \hookrightarrow \mathcal{M}_{n}^{\mathscr{X}}(c)^{s}$. Moreover, each $\mathcal{M}_{n}^{\mathscr{X}}(c)$ is covered by $\mathbf{T w}_{\mathscr{L} / S}(n, \mathcal{O}, c / 2 n)$ in such a way that the fibers are locally $\boldsymbol{\mu}_{n}$-gerbes over 
$\operatorname{Pic}_{X / S}[n]$-torsors, and likewise for the open substacks parametrizing stable objects. This covering restricts to a covering of $\left(f_{*}^{\mathscr{X}} \mathrm{BPGL}_{n}\right)(c)^{s}$ by $\mathbf{T w}_{\mathscr{L} / S}^{s}(n, \mathcal{O}, c / 2 n)^{\text {lf }}$.

In particular, $\mathcal{M}_{n}^{\mathscr{X}}(c)^{s}$ is irreducible, separated, etc. if $\mathbf{T} \mathbf{w}_{\mathscr{L} / S}^{s}(n, \mathbb{O}, c / 2 n)$ has the same property; and $\mathcal{M}_{n}^{\mathscr{X}}(c)^{s}$ has a local property stable for the étale topology if and only if the same is true for $\mathbf{T w}_{\mathscr{Q} / S}^{s}(n, \mathbf{O}, c / 2 n)$ Moreover, there is a virtual fundamental class for $\mathcal{M}_{n}^{\mathscr{X}}(c)^{s}$ if and only if there is one for $\mathbf{T w}_{\mathscr{X} / S}^{s}(n, \mathcal{O}, c / 2 n)$.

Notation 6.1.12. Let $\mathbf{G A z} \mathbf{z}_{\mathscr{L} / S}(n)^{S}$ denote the open substack parametrizing stable generalized Azumaya algebras via the isomorphism $\mathbf{G A z}_{\mathscr{L} / S}(n) \stackrel{\sim}{\rightarrow}\left(\mathcal{M}_{n}^{\mathscr{L}}\right)^{s}$ of Section 5.2.4.

6.2. Structure of moduli of twisted sheaves. The following results show that infinitely many of the spaces $\mathbf{T w}_{\mathscr{Q} / k}^{s}(n, \mathcal{O}, \gamma)$ are nonempty. It is a geometric restatement of the fundamental result of [de Jong 2004] on the period-index problem for Brauer classes over functionf ields of algebraic surfaces.

Lemma 6.2.1. There is a stable locally free $\mathscr{Q}$-twisted sheaf of rank $n$.

A proof of this result may be found in [Lieblich 2008b, Theorem 4.2.2.3] and [Lieblich 2008a, Proposition 5.1.2].

Lemma 6.2.2. Suppose $\mathscr{F}$ is a coherent $\mathscr{X}$-twisted sheaf of rank $n$ with $\operatorname{det} \mathscr{F} \cong 0$ and $\operatorname{deg} c_{2}(\mathscr{F})=\gamma$. For each integer $\ell \geq 0$, there is a (noncanonical) subsheaf $\mathscr{F}_{\ell} \subset \mathscr{F}_{F}$ such that $\operatorname{dim} \mathscr{F}_{F} / \mathscr{F}_{\ell}=0$, det $\mathscr{F}_{\ell} \cong \mathbb{O}$, and $\operatorname{deg} c_{2}\left(\mathscr{F}_{\ell}\right)=\gamma+\ell$. If $\mathscr{F}_{\text {is stable }}$ then so is $\mathscr{F}_{\ell}$.

Proof. By induction, it suffices to construct $\mathscr{F}_{1}$. Choose a point $x \in X(k)$ around which $\mathscr{F}$ is locally free and let $\mathscr{F} \otimes \kappa(x) \rightarrow 2$ be a quotient with geometric fiber of dimension 1. (In other words, given an algebraically closed extension field $L / \kappa(x)$ and a map $\operatorname{Spec} L \rightarrow \mathscr{X} \otimes \kappa(x)$, the pullback of 2 to $\operatorname{Spec} L$ is the sheaf associated to a one-dimensional vector space.) We claim that $\operatorname{deg}\left(c_{2}(2)\right)=-1$, from which the result follows by the multiplicativity of the total Chern polynomial. A proof of the claim uses the Grothendieck-Hirzebruch-Riemann-Roch theorem for representable morphisms of Deligne-Mumford stacks and can be found in the proof of [Lieblich 2007, Lemma 3.2.4.8] (where there is an unfortunate sign error in the statement, even though the proof is correct!).

To deduce stability of $\mathscr{F}_{1}$ from stability of $\mathscr{F}$, first note that the two sheaves agree in codimension 1. Since stability depends on a calculation of degree and this calculation depends only on a sheaf in codimension 1, we see that we need only quantify over saturated subsheaves [Huybrechts and Lehn 1997, Definition 1.1.5], which are determined by their values in codimension 1 . Thus, the criterion determining stability of $\mathscr{F}_{F}$ and $\mathscr{F}_{1}$ quantifies over the same set of subsheaves with the same numerical calculations. 
Corollary 6.2.3. If $\mathbf{T w}_{\mathscr{L} / k}^{s}(n, \mathcal{O}, \gamma)$ is nonempty then so is $\mathbf{T w}_{\mathscr{L} / k}^{s}(n, \mathcal{O}, \gamma+\ell)$ for all integers $\ell \geq 0$.

Here is the fundamental structure theorem concerning these moduli spaces:

Theorem 6.2.4. There exists a constant $C$ such that for all $\gamma \geq C$,

(1) the open substack $\mathbf{T w}_{\mathscr{X} / k}^{s}(n, \mathcal{O}, \gamma)^{l f} \subset \mathbf{T} \mathbf{w}_{\mathscr{X} / k}^{s}(n, \mathcal{O}, \gamma)$ is schematically dense;

(2) $\mathbf{T w}_{\mathscr{L} / k}^{s}(n, \mathcal{O}, \gamma)$ is an irreducible proper normal lci tame Deligne-Mumford stack over $k$ whenever it is nonempty;

(3) it is nonempty for infinitely many $\gamma$.

Proof. The third statement follows immediately from Corollary 6.2.3. For the proof of the first and second, the reader is referred to paragraph 3.2.4.1 (and especially Theorem 3.2.4.11) of [Lieblich 2007].

6.2.5. Since every object of $\mathbf{T w}_{\mathscr{Q} / k}^{s}(n, \mathcal{O}, \gamma)$ is geometrically stable, it is simple (see, for example, Corollary 1.2.8 and Theorem 1.6.6 of [Huybrechts and Lehn 1997]); that is, its automorphisms are simply given by multiplication by scalars (in $\boldsymbol{\mu}_{n}$, since the determinant is trivialized). It follows that $\mathbf{T w}_{\mathscr{L} / k}^{s}(n, \mathcal{O}, \gamma)$ is a $\boldsymbol{\mu}_{n}$-gerbe over its coarse moduli space $\operatorname{Tw}_{\mathscr{Q} / k}^{s}(n, \mathcal{O}, \gamma)$.

\subsection{Consequences for $M_{n}^{\mathscr{X}}$ and $f_{*}^{\mathscr{Q}} \mathrm{BPGL}_{n}$.}

Theorem 6.3.1. There is a constant $D$ such that for all $c \geq D$,

(1) the open substack $f_{*}^{\mathscr{X}} \mathrm{BPGL}_{n}(c)^{s}$ is schematically dense in $\mathcal{M}_{n}^{\mathscr{X}}(c)^{s}$;

(2) $\mathcal{M}_{n}^{\mathscr{X}}(c)^{s}$ is an irreducible proper normal lci tame Deligne-Mumford stack over $k$ whenever it is nonempty;

(3) it is nonempty for infinitely many $c$.

In particular, the open substack $\left(f_{*}^{\mathscr{X}} \mathrm{BPGL}_{n}\right)(c)^{s}$ is irreducible (and nonempty for infinitely many $c$ ).

Proof. The proof follows immediately by combining the covering described at the end of Section 4.2 with Lemma 6.2.1, Lemma 6.2.2, and Theorem 6.2.4.

Recall that "lciq singularities" are by definition finite quotients of lci singularities.

Corollary 6.3.2. For sufficiently large $c$, the coarse moduli space $\left(\mathcal{M}_{n}^{\mathscr{X}}(c)^{s}\right)^{\bmod }$ is an irreducible proper normal algebraic space with lciq singularities.

Proof. Lemma 3.4.2.5 gives rise to a finite morphism

$$
\mathrm{Tw}_{\mathscr{L} / k}^{s}(n, \mathcal{O}, c / 2 n) \rightarrow\left(\mathcal{M}_{n}^{\mathscr{X}}(c)^{s}\right)^{\bmod }
$$


from the coarse space of Paragraph 6.2.5 which is invariant for the natural action of $\operatorname{Pic}_{X / k}[n]$ on $\operatorname{Tw}_{\mathscr{X} / k}^{s}(n, \mathcal{O}, c / 2 n)$ and such that the natural map

$$
\chi: \operatorname{Tw}_{\mathscr{L} / k}^{s}(n, \mathcal{O}, c / 2 n) / \operatorname{Pic}_{X / k}[n] \rightarrow\left(\mathcal{M}_{n}^{\mathscr{X}}(c)^{s}\right)^{\bmod }
$$

is birational. Since the coarse space of a normal tame Deligne-Mumford stack is normal, it follows from Zariski's Main Theorem that $\chi$ is an isomorphism. Since $\mathbf{T w}_{\mathscr{X} / k}^{s}(n, \mathcal{O}, c / 2 n)$ is lci, it follows that $\left(M_{n}^{\mathscr{X}}(c)^{s}\right)^{\mathrm{mod}}$ is lciq.

6.4. Stackification is unnecessary on a surface. Let $f: X \rightarrow S$ be a smooth projective relative surface. We will prove here that pregeneralized Azumaya algebras on $X$ as in Section 5.2 form a stack on $S$.

Given a pregeneralized Azumaya algebra $\mathscr{A}$ on $X$, Lemma 5.2.1.11 produces a $\mathbf{G}_{m}$-gerbe $\mathscr{X}$, an $\mathscr{L}$-twisted sheaf $\mathscr{F}$, and an isomorphism of generalized Azumaya algebras $\mathscr{B}:=\mathbf{R} \pi_{*} \mathbf{R} \mathscr{E}$ nd $(\mathscr{F}) \stackrel{\sim}{\rightarrow} \mathscr{A}$. We will show that in fact $\mathscr{B}$ and $\mathscr{A}$ are isomorphic as pregeneralized Azumaya algebras. We will temporarily call $\mathscr{B}$ the associated twisted derived endomorphism algebra (or TDEA for short).

Proposition 6.4.1. Suppose $f: X \rightarrow S$ is a smooth (possibly nonproper) relative surface over an affine scheme. Any pregeneralized Azumaya algebra A is isomorphic to the associated TDEA in $\mathscr{P} R$. Furthermore, the isomorphisms of two such weak algebras form a sheaf on $S$.

Proof. By standard arguments (for example, [Beylinson et al. 1982, Theorem 3.2.4] or [Abramovich and Polishchuk 2006, Theorem 2.1.9]), it suffices to prove that $\operatorname{Ext}^{-i}(\mathscr{A}, \mathscr{A})=0$ for all $i>0$ (as long as we allow $f: X \rightarrow S$ to be arbirary with the stated hypotheses). From the definition of pregeneralized Azumaya algebra, we know that $\mathscr{A}$ has cohomology only in degrees 0 and 1 , that $\mathscr{H}^{0}(\mathscr{A})$ has totally pure fibers over $S$, and that $\mathscr{H}^{1}(\mathscr{A})$ has support with relative dimension 0 . The natural triangle

$$
\mathscr{H}^{0}(\mathscr{A}) \rightarrow \mathscr{A} \rightarrow \mathscr{H}^{1}(\mathscr{A})[-1] \stackrel{+}{\rightarrow}
$$

gives rise to an exact sequence

$$
\operatorname{Ext}^{-i}\left(\mathscr{H}^{1}(\mathscr{A}), \mathscr{A}[1]\right) \rightarrow \operatorname{Ext}^{-i}(\mathscr{A}, \mathscr{A}) \rightarrow \operatorname{Ext}^{-i}\left(\mathscr{H}^{0}(\mathscr{A}), \mathscr{A}\right) .
$$

The left-hand group fits into an exact sequence

$$
\operatorname{Ext}^{-i}\left(\mathscr{H}^{1}(\mathscr{A}), \mathscr{H}^{0}(\mathscr{A})\right) \rightarrow \operatorname{Ext}^{-i}\left(\mathscr{H}^{1}(\mathscr{A}), \mathscr{A}[1]\right) \rightarrow \operatorname{Ext}^{-i}\left(\mathscr{H}^{1}(\mathscr{A}), \mathscr{H}^{1}(\mathscr{A})\right)
$$

and the right-hand group fits into an exact sequence

$$
\operatorname{Ext}^{-i}\left(\mathscr{H}^{0}(\mathscr{A}), \mathscr{H}^{0}(\mathscr{A})\right) \rightarrow \operatorname{Ext}^{-i}\left(\mathscr{H}^{0}(\mathscr{A}), \mathscr{A}\right) \rightarrow \operatorname{Ext}^{-i-1}\left(\mathscr{H}^{0}(\mathscr{A}), \mathscr{H}^{1}(\mathscr{A})\right) .
$$

(This is simply an explicit description of a certain spectral sequence, which is especially simple because $\mathscr{A}$ has so few cohomology sheaves.) We wish to show that the 
ends of the last two sequences vanish, for which it is enough to show (using the local-to-global Ext-spectral sequence) that the Ext-sheaves $\mathscr{E} x t^{*}\left(\mathscr{H}^{*}(\mathscr{A}), \mathscr{H}^{*}(\mathscr{A})\right)$ vanish for appropriate indices. But there are no negative Ext-groups for modules over a ring. This completes the proof.

Remark 6.4.2. When $X / S$ is quasiprojective, one can also give an explicit proof of Proposition 6.4.1 (which does not rely on [Beĭlinson et al. 1982]) using resolutions by sums of powers of $O(1)$.

6.5. Deformation theory and the virtual fundamental class. Let $k$ be an algebraically closed field and $X / k$ a smooth projective surface over $k$. Fix a $\boldsymbol{\mu}_{n}$-gerbe $\mathscr{X} \rightarrow X$ with $n$ invertible in $k$.

6.5.1. Perfect obstruction theory for twisted sheaves. Let $\mathscr{F}$ be the universal $\mathscr{L}$ twisted sheaf on $\mathscr{X} \times \mathbf{T w}_{\mathscr{L} / k}^{s}(n, \mathcal{O})$. Write $p$ (resp. $q$ ) for the projection of $\mathscr{X} \times$ $\mathbf{T} \mathbf{w}_{\mathscr{X} / k}^{s}(n, \mathcal{O})$ to $\mathscr{X}\left(\operatorname{resp} . \mathbf{T} \mathbf{w}_{\mathscr{L} / k}^{s}(n, \mathcal{O})\right)$. Recall that there is a natural isomorphism

$$
L_{\mathscr{L} \times \mathbf{T} \mathbf{w}_{\mathscr{C} / k}^{s}(n, \mathcal{O})} \cong \mathbf{L} p^{*} L_{\mathscr{L}} \oplus \mathbf{L} q^{*} L_{\mathbf{T} \mathbf{w}_{\mathscr{C} / k}^{s}(n, \mathcal{O})},
$$

and that there is an isomorphism of functors (coming from Grothendieck duality for $q$ )

$$
\mathbf{L} q^{*} \cong \mathbf{L} q^{!} \stackrel{\mathbf{L}}{\otimes} \mathbf{L} p^{*} \omega_{X}^{\vee}[-2] .
$$

The Atiyah class

$$
\mathscr{F} \rightarrow L_{\mathscr{X}} \times \mathbf{T w}_{\mathscr{W} / k}^{s}(n, \mathcal{O}) \stackrel{\mathbf{L}}{\otimes} \mathscr{F}[1]
$$

yields by projection a map

$$
\mathscr{F} \rightarrow \mathbf{L} q^{*} L_{\mathbf{T w}_{\mathscr{X} / k}^{s}(n, \mathcal{O})}[1] \stackrel{\mathbf{L}}{\otimes} \mathscr{F} .
$$

Since $\mathscr{F}$ is perfect, this is equivalent (by the cher à Cartan isomorphism) to a map

$$
\mathbf{R}_{\mathscr{E}}^{\mathrm{E}} \mathrm{nd}(\mathscr{F}) \rightarrow \mathbf{L} q^{*} L_{\mathbf{T w}_{\mathscr{C} / k}^{s}(n, \mathcal{O})}[1]
$$

yielding a map

$$
\mathbf{R}^{\mathscr{E n d}}(\mathscr{F}) \rightarrow \mathbf{L} q^{!} L_{\mathbf{T}} \mathbf{w}_{\mathscr{C} / k}^{s}(n, \mathcal{O})[-1] \stackrel{\mathbf{L}}{\otimes} \mathbf{L} p^{*} \omega_{X}^{\vee} .
$$

Applying Grothendieck duality yields a morphism

$$
\mathfrak{b}: \mathbf{R} q_{*} \mathbf{R} \mathscr{H o m}\left(\mathscr{F}, \mathbf{L} p^{*} \omega_{X} \stackrel{\mathbf{L}}{\otimes} \mathscr{F}\right) \rightarrow L_{\mathbf{T} \mathbf{w}_{\mathscr{x} / k}^{s}(n, \mathcal{O})}[-1]
$$

and restriction to the traceless part finally yields a morphism

$$
\mathfrak{b}_{0}: \mathbf{R} q_{*} \mathbf{R} \mathscr{H} \operatorname{lom}\left(\mathscr{F}, \mathbf{L} p^{*} \omega_{X} \stackrel{\mathbf{L}}{\otimes} \mathscr{F}\right)_{0} \rightarrow L_{\mathbf{T} \mathbf{w}_{\mathscr{Q} / k}^{s}(n, \mathcal{O})}[-1]
$$

Proposition 6.5.1.1. The shifted map $\mathfrak{b}_{0}[1]$ gives a perfect obstruction theory for $\mathbf{T w}_{\mathscr{X} / k}^{s}(n, 0)$. 
Proof. There are three things to check: that the complex $\mathbf{R} q_{*} \mathbf{R} \mathscr{H} \operatorname{lom}\left(\mathscr{F}, \mathbf{L} p^{*} \omega_{X} \stackrel{\mathbf{L}}{\otimes}\right.$ $\mathscr{F})_{0}$ is perfect of amplitude $[0,1]$, that $\mathfrak{b}_{0}$ induces an isomorphism on $\mathrm{H}^{1}$ sheaves, and that $\mathfrak{b}_{0}$ induces a surjection on $\mathrm{H}^{0}$ sheaves. The first assertion follows from the compatibility of the formation of the complex with base change on $\mathbf{T w}_{\mathscr{Q} / k}^{s}(n, \mathbb{O})$ (which shows that it is perfect, as its fibers are bounded complexes on regular schemes) and the fact that the fibers of $\mathbf{R}^{2} q_{*} \mathbf{R} \mathscr{H}$ om $\left(\mathscr{F}, \mathbf{L} p^{*} \omega_{X} \stackrel{\mathbf{L}}{\otimes} \mathscr{F}\right)_{0}$ over geometric points of $\mathbf{T w}_{\mathscr{Q} / k}^{s}(n, \mathbb{O})$ compute (by Serre duality) the traceless endomorphisms of stable sheaves, which must be trivial (so that the amplitude is as claimed). The other two assertions will follow from Illusie's theory. We already know (thanks to Illusie) that deformations and obstructions are governed by Atiyah classes; we will describe how this allows us to show that $\mathfrak{b}_{0}$ gives a perfect obstruction theory.

Since both the domain and codomain of $\mathfrak{b}_{0}$ have no cohomology above degree 1 , to show that $\mathfrak{b}_{0}$ induces an isomorphism on $\mathrm{H}^{1}$ sheaves, it suffices to show this after base change to geometric points of $\mathbf{T w}_{\mathscr{X} / k}^{s}(n, \mathbb{O})$. Given a geometric point $x \rightarrow \mathbf{T w}_{\mathscr{L} / k}^{s}(n, \mathcal{O})$ corresponding to a twisted sheaf on $\mathscr{L} \otimes \kappa(x)$, we know that $\operatorname{Hom}\left(L_{\mathbf{T w}_{\mathscr{X} / k}^{s}(n, \mathcal{O})}, \kappa(x)\right)$ is naturally identified with the space $T$ of first order determinant-preserving deformations of $F$ over $\kappa(x)[\varepsilon]$. Moreover, by the functoriality of our construction with respect to base change on $\mathbf{T w}_{\mathscr{O} / k}^{s}(n, \mathcal{O})$, the map $\mathfrak{b}_{0}$ is the Serre dual of the Kodaira-Spencer map $T \rightarrow \operatorname{Ext}^{1}(F, F)_{0}$ (see, for example, [Huybrechts and Lehn 1997, Example 10.1.9]). This is well-known to give an isomorphism (for example, [Huybrechts and Lehn 1997, Section 10.2]).

To show that $\mathrm{H}^{0}\left(\mathfrak{b}_{0}\right)$ is surjective, we may proceed as follows. Recall from [Illusie 1971, IV.3.1.8] that if $A \rightarrow A_{0}$ is a small extension of $B$-algebras with kernel $I$ in a topos and $M$ is an $A_{0}$-module, then one can find the obstruction to deforming $M$ to an $A$-module as the composition

$$
M \rightarrow L_{A_{0} / B} \stackrel{\mathbf{L}}{\otimes} M[1] \rightarrow I \stackrel{\mathbf{L}}{\otimes} M[2] \rightarrow I \otimes M[2],
$$

where the first map is the Atiyah class of $M$ with respect to $A_{0} / B$, the second map comes from the morphism $L_{A_{0} / B} \rightarrow I$ parametrizing the class of the extension $A \rightarrow A_{0}$, and the third map is the natural augmentation onto the zeroth cohomology module. To apply this to our case, consider a situation

$$
\operatorname{Spec} B \leftarrow \operatorname{Spec} B_{0} \rightarrow \mathbf{T w}_{\mathscr{X} / k}^{s}(n, \mathcal{O})
$$

with $B \rightarrow B_{0}$ a small extension of strictly Henselian local rings and kernel annihilated by the maximal ideal of $B$. We let $A$ be the structure sheaf of $\mathscr{X} \times \operatorname{Spec} B$ and $A_{0}$ that of $\mathscr{L} \times \operatorname{Spec} B_{0}=\mathscr{X} \times \operatorname{Spec} B \times \operatorname{Spec} B \operatorname{Spec} B_{0}$. Thus, we have that

$$
L_{A_{0} / B}=\mathbf{L} p^{*} L_{\mathscr{C}} \oplus \mathbf{L} q^{*} L_{B_{0} / B}
$$


Moreover, it is clear that the morphism $L_{A_{0} / B} \rightarrow \mathscr{O}_{\mathscr{X}} \otimes I[1]$ parametrizing the extension $A \rightarrow A_{0}$ is given by the map

$$
L_{A_{0} / B} \rightarrow \mathbf{L} q^{*} L_{B_{0} / B} \rightarrow \mathbf{L} q^{*} I[1] .
$$

By functoriality, composing the Atiyah class with the natural map

$$
\left.L_{\mathbf{T w}_{\mathscr{Q} / k}^{s}(n, \mathcal{O})}\right|_{B_{0}} \rightarrow L_{B_{0} / B}
$$

gives rise to the map

$$
\mathscr{F} \rightarrow \mathbf{L} q^{*} L_{B_{0} / B} \stackrel{\mathbf{L}}{\otimes} \mathscr{F}[1]
$$

associated to the projection of the Atiyah class. Thus, we find that the obstruction to deforming $\mathscr{F}$ over $B$ is the element corresponding by Serre duality to the composition

$$
\left.\left.\mathbf{R} q_{*} \mathbf{R} \mathscr{H} \operatorname{lom}\left(\mathscr{F}, \mathbf{L} q^{*} \omega \stackrel{\mathbf{L}}{\otimes} \mathscr{F}\right)\right|_{B_{0}} \rightarrow L_{\mathbf{T w}_{\mathscr{C} / k}(n, \mathcal{O})}[-1]\right|_{B_{0}} \rightarrow L_{B_{0} / B}[-1] \rightarrow \kappa .
$$

On the other hand, the last two arrows give precisely the obstruction to extending the map $\operatorname{Spec} B_{0} \rightarrow \mathbf{T w}_{\mathscr{X} / k}(n, \mathcal{O})$ to a map $\operatorname{Spec} B \rightarrow \mathbf{T w}_{\mathscr{X} / k}(n, \mathcal{O})$. Thus, the entire composition is trivial if and only if the composition of the last two maps is trivial. Since any map $L_{\mathbf{T w}_{\mathscr{K} / k}(n, \mathcal{O})} \rightarrow \kappa[1]$ factors through some deformation situation $B \rightarrow B_{0}$, this shows that $\mathrm{H}^{0}(\mathfrak{b})$ is surjective. Using the fact that $n$ is invertible in $k$, and thus the existence of a splitting trace map, it is easy to see that the canonical obstruction given here actually lies in the traceless part of $\operatorname{Ext}^{2}(\mathscr{F}, \mathscr{F})$; this shows that in fact $\mathrm{H}^{0}\left(\mathfrak{b}_{0}\right)$ is surjective, as desired.

The deformation theory described here has a concrete form: given a generalized Azumaya algebra $\mathbf{R}^{\mathscr{E}} \mathrm{nd}(\mathscr{F})$ on $X$, the first-order infinitesimal deformations form a pseudo-torsor under the hypercohomology $\mathbf{H}^{1}\left(X, \mathbf{R}^{\mathscr{E}} \mathrm{nd}(\mathscr{F})_{0}\right)$, while there is naturally a class in $\mathbf{H}^{2}\left(X, \mathbf{R}^{\complement} \mathbb{E} d(\mathscr{F})_{0}\right)$ giving the obstruction to deforming $\mathscr{F}$. When $\mathscr{F}$ is locally free, so that $\mathbf{R}_{\mathscr{C}} \mathrm{nd}(\mathscr{F}) \cong A$ is an Azumaya algebra, we recover the well-known fact that $\mathrm{H}^{1}\left(X, A_{0}\right)$ parametrizes deformations of $A$, while $\mathrm{H}^{2}\left(X, A_{0}\right)$ receives obstructions. If $A \cong \mathscr{H o m}(\mathscr{V})$ is the sheaf of endomorphisms of a locally free sheaf on $X$ with trivial(ized) determinant, our general machine simply says that the deformation and obstruction theory of the algebra $A$ is the same as the deformation and obstruction theory of $\mathscr{V}$ as a locally free sheaf with trivialized determinant. (This similarly describes the deformation theory of a twisted sheaf with trivialized determinant.)

6.5.2. The virtual fundamental class of $\mathbf{G A z}_{\mathscr{X} / k}(n)^{s}$. By construction, $\mathbf{G A z}_{\mathscr{L} / k}(n)^{s}$ is a subfibered category of the fibered category of weak algebras on $X_{\text {rét }}$. As such, there is a universal generalized Azumaya algebra $\mathscr{A}$ on $X \times \mathbf{G A z}_{\mathscr{L} / k}(n)^{s}$ whose 
fibers over the moduli space have cohomology class [थ]. If

$$
\pi: \text { 甲 } \rightarrow X \times \mathbf{G A z}_{\mathscr{X} / k}(n)^{s}
$$

is the gerbe of trivialized trivializations of $\mathscr{A}$, then $\mathscr{A} \cong \mathbf{R} \pi_{*} \mathbf{R} \mathscr{E} d(\mathscr{F})$ for some y-twisted sheaf $\mathscr{F}$. Moreover, the covering $\mathbf{T w}_{\mathscr{X} / k}^{s}(n, \mathcal{O}) \rightarrow \mathbf{G A z} \mathscr{\mathscr { L } / k}(n)^{s}$ gives rise to an isomorphism

$$
\rho: \mathscr{Y} \times_{X \times \mathbf{G A z} \mathscr{\mathscr { K }} / k}(n)^{s} X \times \mathbf{T w}_{\mathscr{X} / k}^{s}(n, \mathcal{O}) \stackrel{\sim}{\rightarrow} \mathscr{X} \times \mathbf{T} \mathbf{w}_{\mathscr{L} / k}^{s}
$$

as well as an isomorphism $\mathscr{G} \rightarrow \rho^{*} \mathscr{F}$, where $\mathscr{G}$ is the universal twisted sheaf on $\mathscr{X} \times \mathbf{T w}_{\mathscr{X} / k}^{s}(n, \mathcal{O})$. There results from this a natural isomorphism of weak algebras

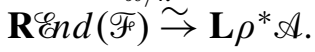

Letting $\mathscr{A}_{0} \subset \mathscr{A}$ be the traceless part, there is an induced isomorphism

$$
\mathbf{R}_{\mathscr{E}} \operatorname{nd}(\mathscr{F})_{0} \stackrel{\sim}{\rightarrow} \mathbf{L} \rho^{*} \mathscr{A}_{0}
$$

Applying Proposition 3.4.3.7 and Proposition 6.5.1.1, we conclude that there is a perfect obstruction theory $\mathscr{A}_{0} \rightarrow L_{\mathbf{G A z}_{\mathscr{E} / k}(n)^{s}}$, giving rise to a virtual fundamental class on $\mathbf{G A z}_{\mathscr{L} / k}(n)^{s}$.

\subsection{A potential application: numerical invariants of division algebras over func-} tion fields. Suppose that the cohomology class $\alpha$ of $\mathscr{X}$ in $\mathrm{H}^{2}\left(X, \mathbf{G}_{m}\right)$ has order $n$. If $\mathscr{A}$ is an Azumaya algebra of degree $n$ with cohomology class $\alpha$, then the generic fiber of $\mathscr{A}$ must be a finite dimensional central division algebra $D$ over the function field $k(X)$. In this case, we have an especially nice description of the stable locus.

Lemma 6.6.1. When [थ] has order $n$ in $\mathrm{H}^{2}\left(X, \mathbf{G}_{m}\right)$, any $\mathrm{PGL}_{n}$-torsor $T$ with class $\alpha$ is stable.

Proof. Indeed, if $\mathscr{A}$ is the Azumaya algebra associated to $T$ then any nonzero right ideal must have rank $n^{2}$, since the generic fiber of $\mathscr{A}$ is a division algebra.

Thus, $\mathbf{G A z}_{\mathscr{L} / k}(n)^{s}$ is a proper Deligne-Mumford stack which carries a virtual fundamental class, as described in Section 6.5.2. The following question was asked by de Jong.

Question 6.6.2. Does the virtual class $\left[\mathbf{G A z}_{\mathscr{L} / k}(n)^{s}\right]^{\mathrm{vir}}$ lead to any new numerical invariants attached to $D$ ?

Via Proposition 3.4.3.7, any invariants coming from $\left[\mathbf{G A} \mathbf{z}_{\mathscr{L} / k}(n)^{s}\right]^{\mathrm{vir}}$ will be closely related to similar numbers attached to $\left[\mathbf{T w}_{\mathscr{L} / k}^{s}(n, O)^{s}\right]^{\mathrm{vir}}$. One might expect the latter invariants to be related to Donaldson invariants.

One interesting direct comparison might arise as follows: suppose given a family of surfaces $\mathscr{X} \rightarrow S$ and a class $\alpha \in \mathrm{H}^{2}\left(\mathscr{X}, \boldsymbol{\mu}_{n}\right)$ with $n$ invertible on the base, such that there are two geometric points $0,1 \rightarrow S$ such that $\left.\alpha\right|_{\mathscr{L}_{0}}$ has order $n$ in $\mathrm{H}^{2}\left(\mathscr{C}_{0}, \mathbf{G}_{m}\right)$ and $\left.\alpha\right|_{\mathscr{L}_{1}}$ vanishes in $\mathrm{H}^{2}\left(\mathscr{X}_{1}, \mathbf{G}_{m}\right)$. (This happens whenever there is jumping in 
the rank of the Néron-Severi group in the family.) Assuming one could prove deformation invariance of whatever invariants one eventually defines, one would then be able to give a direct comparision between the division-algebra invariants attached to $\mathscr{X}_{0}$ and the classical invariants attached to $\mathscr{X}_{1}$.

\section{Acknowledgments}

The results of this paper are an extension of part of the author's Ph.D. thesis. He would like to thank his adviser, Aise Johan de Jong, for many helpful conversations. The author also greatly benefitted from contact with Dan Abramovich, Jean-Louis Colliot-Thélène, Tomás Gómez, Daniel Huybrechts, Martin Olsson, and Kōta Yoshioka during various stages of this project. He thanks the referee for useful comments.

\section{References}

[Abramovich and Polishchuk 2006] D. Abramovich and A. Polishchuk, "Sheaves of $t$-structures and valuative criteria for stable complexes", J. Reine Angew. Math. 590 (2006), 89-130. MR 2007g: 14014 Zbl 1093.14026

[Abramovich et al. 2003] D. Abramovich, A. Corti, and A. Vistoli, "Twisted bundles and admissible covers”, Comm. Algebra 31:8 (2003), 3547-3618. MR 2005b:14049 Zbl 1077.14034

[Artin 1973] M. Artin, Théorèmes de représentabilité pour les espaces algébriques, Séminaire de Mathématiques Supérieures 44, Les Presses de l'Université de Montréal, Montreal, Que., 1973. MR 53 \#10794 Zbl 0323.14001

[Artin 1974] M. Artin, "Versal deformations and algebraic stacks", Invent. Math. 27 (1974), 165189. MR 53 \#2945 Zbl 0317.14001

[Bělinson et al. 1982] A. A. Beǔlinson, J. Bernstein, and P. Deligne, "Faisceaux pervers", pp. 5-171 in Analysis and topology on singular spaces, I (Luminy, 1981), Astérisque 100, Soc. Math. France, Paris, 1982. MR 86g:32015 Zbl 0536.14011

[Căldăraru 2002a] A. Căldăraru, "Nonfine moduli spaces of sheaves on K3 surfaces", Int. Math. Res. Not. 20 (2002), 1027-1056. MR 2003b:14017 Zbl 1057.14020

[Căldăraru 2002b] A. Căldăraru, "Derived categories of twisted sheaves on elliptic threefolds", $J$. Reine Angew. Math. 544 (2002), 161-179. MR 2003a:14022 Zbl 0995.14012

[Giraud 1971] J. Giraud, Cohomologie non abélienne, vol. 179, Die Grundlehren der mathematischen Wissenschaften, Springer, Berlin, 1971. MR 49 \#8992 Zbl 0226.14011

[Gómez and Sols 2005] T. Gómez and I. Sols, "Moduli space of principal sheaves over projective varieties”, Ann. of Math. (2) 161:2 (2005), 1037-1092. MR 2006e:14014 Zbl 1079.14018

[Grothendieck 1961] A. Grothendieck, "Éléments de géométrie algébrique. III. Étude cohomologique des faisceaux cohérents. I”, Inst. Hautes Études Sci. Publ. Math. 11 (1961), 167. MR 29 \#1209 Zbl 0118.36206

[Grothendieck 1971] A. Grothendieck (editor), Revêtements étales et groupe fondamental, Lecture Notes in Mathematics 224, Springer, Berlin, 1971. Séminaire de Géométrie Algébrique du Bois Marie 1960-1961 (SGA 1), Dirigé par Alexandre Grothendieck. Augmenté de deux exposés de M. Raynaud. MR 50 \#7129 Zbl 1039.14001 
[Hartshorne 1966] R. Hartshorne, Residues and duality, Lecture Notes in Mathematics 20, Springer, Berlin, 1966. MR 36 \#5145 Zbl 0212.26101

[Heinloth 2005] J. Heinloth, "Twisted Chern classes and $\mathbb{G}_{m}$-gerbes", C. R. Math. Acad. Sci. Paris 341:10 (2005), 623-626. MR 2006h:14008 Zbl 1091.14005

[Hoffmann and Stuhler 2005] N. Hoffmann and U. Stuhler, "Moduli schemes of generically simple Azumaya modules”, Doc. Math. 10 (2005), 369-389. MR 2006j:14060 Zbl 1092.14051

[Huybrechts and Lehn 1997] D. Huybrechts and M. Lehn, The geometry of moduli spaces of sheaves, Aspects of Mathematics E31, Friedr. Vieweg \& Sohn, Braunschweig, 1997. MR 98g:14012 Zbl 0872.14002

[Hyeon 2002] D. Hyeon, "Principal bundles over a projective scheme", Trans. Amer. Math. Soc. 354:5 (2002), 1899-1908. MR 2003d:14013 Zbl 1014.14002

[Illusie 1971] L. Illusie, Complexe cotangent et déformations. I, Lecture Notes in Mathematics 239, Springer, Berlin, 1971. MR 58 \#10886a Zbl 0224.13014

[de Jong 2004] A. J. de Jong, "The period-index problem for the Brauer group of an algebraic surface”, Duke Math. J. 123:1 (2004), 71-94. MR 2005e:14025 Zbl 1060.14025

[Knudsen and Mumford 1976] F. F. Knudsen and D. Mumford, "The projectivity of the moduli space of stable curves. I. Preliminaries on "det" and "Div"”, Math. Scand. 39:1 (1976), 19-55. MR 55 \#10465 Zbl 0343.14008

[Kovács and Lieblich 2006] S. Kovács and M. Lieblich, "Boundedness of families of canonically polarized manifolds: A higher dimensional analogue of Shafarevich's conjecture”, preprint, 2006. arXiv math/0611672

[Langer 2005] A. Langer, "Semistable principal G-bundles in positive characteristic", Duke Math. J. 128:3 (2005), 511-540. MR 2006b:14016 Zbl 1081.14018

[Laumon and Moret-Bailly 2000] G. Laumon and L. Moret-Bailly, Champs algébriques, Ergebnisse der Mathematik und ihrer Grenzgebiete. 3. Folge. 39, Springer, Berlin, 2000. MR 2001f:14006 Zbl 0945.14005

[Lieblich 2006a] M. Lieblich, "Moduli of complexes on a proper morphism", J. Algebraic Geom. 15:1 (2006), 175-206. MR 2006f:14009 Zbl 1085.14015

[Lieblich 2006b] M. Lieblich, "Remarks on the stack of coherent algebras", Int. Math. Res. Not. (2006), Art. ID 75273, 12. MR 2008c:14022 Zbl 1108.14003

[Lieblich 2007] M. Lieblich, "Moduli of twisted sheaves", Duke Math. J. 138:1 (2007), 23-118. MR 2008d:14018 Zbl 1122.14012

[Lieblich 2008a] M. Lieblich, "Moduli of twisted orbifold sheaves", preprint, 2008. submitted for publ. arXiv 0803.3332

[Lieblich 2008b] M. Lieblich, “Twisted sheaves and the period-index problem”, Compos. Math. 144:1 (2008), 1-31. MR 2009b:14033 Zbl 1133.14018

[Matsumura 1989] H. Matsumura, Commutative ring theory, 2nd ed., Cambridge Studies in Advanced Mathematics 8, Cambridge University Press, 1989. MR 90i:13001 Zbl 0603.13001

[Romagny 2005] M. Romagny, "Group actions on stacks and applications", Michigan Math. J. 53:1 (2005), 209-236. MR 2005m:14005 Zbl 1100.14001

[Schmitt 2002] A. H. W. Schmitt, "Singular principal bundles over higher-dimensional manifolds and their moduli spaces”, Int. Math. Res. Not. 23 (2002), 1183-1209. MR 2003e:14007 Zbl 1034. 14017

[Schmitt 2004] A. H. W. Schmitt, "A closer look at semistability for singular principal bundles", Int. Math. Res. Not. 62 (2004), 3327-3366. MR 2005h:14105 Zbl 1093.14507 
[Simpson 1994] C. T. Simpson, "Moduli of representations of the fundamental group of a smooth projective variety. I", Inst. Hautes Études Sci. Publ. Math. 79 (1994), 47-129. MR 96e:14012 Zbl 0891.14005

[Taubes 1989] C. H. Taubes, "The stable topology of self-dual moduli spaces", J. Differential Geom. 29:1 (1989), 163-230. MR 90f:58023 Zbl 0669.58005

[Wang 2002] X. Wang, "Balance point and stability of vector bundles over a projective manifold", Math. Res. Lett. 9:2-3 (2002), 393-411. MR 2004f:32034 Zbl 1011.32016

[Wang 2005] X. Wang, "Canonical metrics on stable vector bundles", Comm. Anal. Geom. 13:2 (2005), 253-285. MR 2006b:32031 Zbl 1093.32008

[Yoshioka 2006] K. Yoshioka, "Moduli spaces of twisted sheaves on a projective variety", pp. 1-30 in Moduli spaces and arithmetic geometry, edited by S. Mukai et al., Adv. Stud. Pure Math. 45, Math. Soc. Japan, Tokyo, 2006. MR 2008f:14024 Zbl 1118.14013

Communicated by Jean-Louis Colliot-Thélène

Received 2008-10-19 Revised 2009-05-24 Accepted 2009-06-25

lieblich@math.washington.edu Department of Mathematics, Princeton University, Fine Hall, Washington Road, Princeton, NJ 08544-1000, United States

Current address: Department of Mathematics, University of Washington, Box 354350, Seattle, WA 98195, United States 